\title{
A series-resonant inverter with extended topology and pulse- density-modulation control for induction heating applications
}

\author{
Pavlo Herasymenko, Volodymyr Pavlovskyi, Oleg Yurchenko \\ Department of transistor converters, Institute of Electrodynamics, Kyiv, Ukraine
}

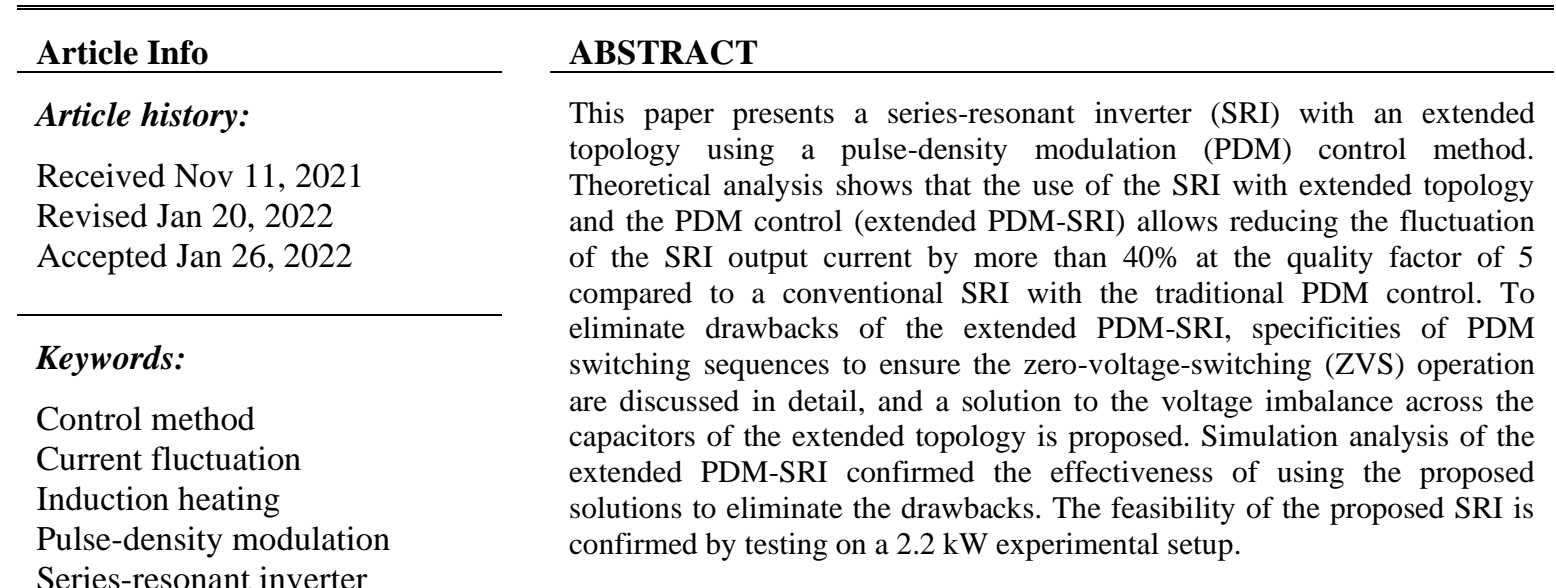

This is an open access article under the CC BY-SA license.

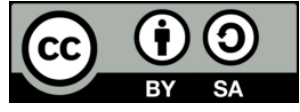

\section{Corresponding Author:}

Pavlo Herasymenko

Department of Transistor Converters, Institute of Electrodynamics

03057, 56 Peremohy avenue, Kyiv, Ukraine

Email: herasymenko@ieee.org

\section{INTRODUCTION}

Recently, the pulse-density modulation (PDM) control method [1]-[6] has been widely applied as a transistor converter current control method for many applications such as induction heating [1], [2], [7]-[10] and many others [3]-[6], [11]-[13]. In comparison with other control methods, such as pulse-width modulation, pulse-frequency modulation (PFM), or phase-shift control, the PDM control in the converters of induction heating equipment based on a series-resonant inverter (SRI) allows SRI transistors to operate with zero-voltage switching (ZVS) and quasi-zero-current switching (quasi-ZCS). As a result, high-frequency switching operation can be performed, switching losses and a surge voltage can be reduced, and the structure of induction heating equipment can be simplified. Despite the advantages of this method for the SRI, using the PDM control also has disadvantages; the main is that the amplitude of the SRI output current fluctuates. The fluctuation influences the maximum current through the SRI transistors, the maximum voltage across the resonant capacitor, and the required dead-time between the control signals of the SRI transistors. And the lower the value of the quality factor of a resonant circuit is, the higher the fluctuation is.

The basic idea of the PDM control method is that the SRI produces injection and free-wheeling intervals [7]-[9]. The output current is regulated by varying the duration of these intervals. In the traditional PDM control the duration of the injection and free-wheeling intervals is an integer multiple of the SRI output voltage period. The same pulse density and different amplitude fluctuations can be achieved with regular and irregular PDM controls [14]-[16]. The use of the irregular PDM control compared to the regular one allows to obtain lower fluctuations, but they are still significant. 
In order to reduce the amplitude fluctuations and make the PDM control method more suitable for the SRI with a low value of a load quality factor, various enhanced PDM controls have been proposed [1][3], [6], [17], [18]. The general idea of these controls is that the duration of both the injection and freewheeling intervals or just the injection interval is an integer multiple of the half-period of the SRI output voltage. When both the injection and free-wheeling intervals are integers multiple of half-period of the SRI output voltage, the voltage contains a direct current (DC) component [1]-[3]. This is not very suitable if a matching transformer is required, because a magnetizing current appears in the matching transformer due to the DC component. To avoid the influence of this component, Esteve et al. [1] used a blocking capacitor. But there also appears an unbalance in the inverter transistors' switching loss. To avoid this unbalance, both halfbridges must operate alternatively during each enhanced PDM period. The switching sequence of the enhanced PDM can be further evenly distributed; this results in an additional reduction in current fluctuations [6], [17]. In both cases, the sign of the DC-component will alternate depending on the voltage sequence; as a result, the blocking capacitor will not be effective, and as a consequence, the matching transformer must be rated for a frequency lower than the operating one. When only the injection interval is an integer multiple of half-period of the voltage, the voltage does not contain the DC component. Still, fluctuations are not significantly lower compared to the traditional PDM control method [18].

Combined control methods based on the PDM control have been proposed [19]-[22]. A hybrid control method based on the PDM and PFM controls has been proposed for corona discharge treatment [19]. This method uses the regular PDM control, so if being applied in SRIs for induction heating applications, the amplitude fluctuations of the current will be significant. Shen et al. in [20], Namadmalan [21], the authors used the phase-shift and PDM controls for SRIs, where former acts between PDM patterns to provide more continuous/smooth power regulation. However, the mentioned method does not allow reducing the amplitude fluctuations. Herasymenko in [22], another combined control method based on the phase-shift and PDM controls is proposed. In this method, the current regulation for pulse density in the range from 0.5 to 1 is provided by only the traditional PDM control, and in the range from 0 to 0.5 - by the phase-shift control and the constant pulse density (equal to 0.5 ) of the PDM pattern. Disadvantages of this approach are that the use of the phase-shift control method increases the switch losses of the SRI transistors and besides, the amplitude fluctuations in the range from 0 to 0.5 are not reduced.

Another control method of the SRI output current, close to the PDM control method, is the odd ( $3^{\text {rd }}$ or $5^{\text {th }}$ ) harmonic operation [21], [23], [24]. The advantage of this method is lower commutation losses in the SRI transistors. But in this case, the frequency of the SRI output voltage is three, five, or more times less than the resonance one. Thus, the size of the matching transformer is significantly larger compared to the case when the PDM control method is used. In addition, the amplitude fluctuations are larger compared to the irregular PDM control.

One more approach to reducing the fluctuation is the use of a modular converter, which is based on series or parallel connection of inverters, with the interleaved or stepped PDM control methods [3], [25][28]. In the case of using the interleaved PDM control, each channel of the converter has the same PDM pattern, but there is a time-shift between them, which influences the fluctuation [25]-[28]. In the case of using the stepped PDM control, the modulation begins to act only in one of the converter channels, and the modulation signals of other channels are 0 and/or 1 [28]. Compared to the single-channel SRI, the modular converter must have at least two channels, so more transistors are required; the constructions of the modular converter and the matching transformer are more complex, larger in dimensions, and finally, the modular converter costs more.

The SRI with extended topology and the PDM control method (extended PDM-SRI) has been presented in our primary work [29]. The present paper expands on previous research and proposes solutions to problems associated with the drawbacks mentioned in the primary work. The main contributions of this paper are summarized is being as: 1) This article improves and enhances the theoretical analysis of the extended PDM-SRI, in particular through a deeper discussion of the amplitude fluctuations of the SRI output current. 2) Drawbacks of diodes reverse-recovery problems and voltage imbalance across capacitors mentioned in [29] are discussed, and solutions of these problems are proposed. 3) Simulation analysis of the extended PDM-SRI was carried out to verify the proposed solutions. The validity of the theoretical and simulation analysis is verified by a $2.2 \mathrm{~kW}$ experimental setup of the discussed extended PDM-SRI.

\section{THEORETICAL BASIS}

\subsection{Induction heating equipment configuration}

A typical configuration of induction heating equipment consists of a diode full-bridge rectifier, a DC-filter, a transistor inverter, a matching transformer, a control system, and a current transformer (CT) Figure 1. A load (an induction coil with a workpiece) of an induction heating system can be represented by the series-equivalent circuit model with an equivalent inductor $L_{e q}$ and an equivalent resistor $R_{e q}$; the model

A series-resonant inverter with extended topology and pulse-density-modulation ... (Pavlo Herasymenko) 
represents inductances of the workpiece, the induction coil, the gap, and resistances of the workpiece and the induction coil [30], [31]. The values of these parameters depend on geometries and materials of the induction coil and workpiece, and the operating frequency of the SRI. To obtain a series-resonant circuit, a capacitor $C_{r}$ is usually connected in series with the secondary winding of the matching transformer and the load.

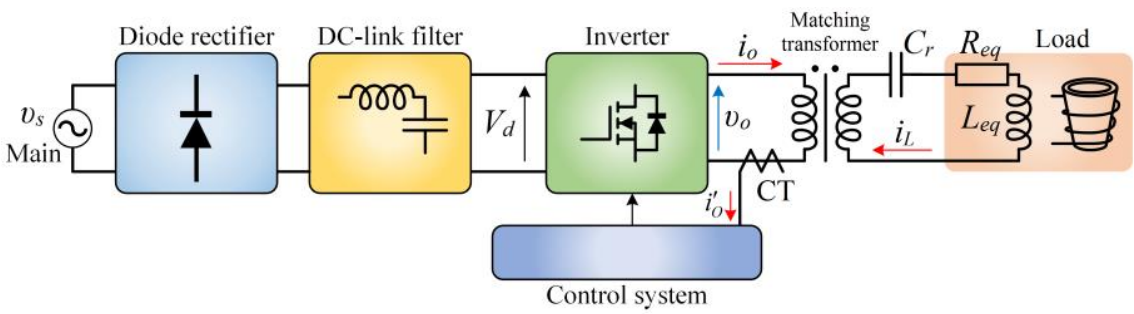

Figure 1. Typical configuration of induction heating equipment

Typically, the PDM control method is used in inverters with the full-bridge topology, because with this topology it is possible to obtain the injection and free-wheeling intervals [1], [2], [7]-[9]. This control method is also suitable in inverters with the half-bridge topology [10]. In this case the PDM-SRI produces two intervals: an injection one with a square-wave ac-voltage equal to half the SRI input voltage, and a rejection one, when the resonant circuit energy is transmitted to the capacitors of the half-bridge topology. As a consequence, the amplitude fluctuations of the SRI output current are higher.

\subsection{Pulse-density modulation-series-resonant inverter with extended topology}

To reduce the amplitude fluctuations of the PDM-SRI output current $i_{O}$ it is expedient to combine operation modes of full-bridge and half-bridge inverters [29]. For this purpose, the extended inverter topology is used, which combines topologies of full-bridge and half-bridge inverters [29], [32], [33]. The extended inverter topology consists of switching devices $\left(\mathrm{Q}_{1}-\mathrm{Q}_{4}\right)$ of the full-bridge topology, two capacitors $\left(C_{1}\right.$ and $\left.C_{2}\right)$ of equal values of the half-bridge topology, and an additional bi-directional switch Figure 2 . The capacitors are series-connected across the DC source $\left(V_{d}\right)$, and the SRI input voltage $V_{d}$ is split by these capacitors into two equal sources with a voltage of $V_{d} / 2$. The bi-directional switch is implemented with two back-to-back connected transistors $\left(\mathrm{Q}_{5}\right.$ and $\left.\mathrm{Q}_{6}\right)$ in a typical source configuration. This switch is connected between the center tap of the series-connected capacitors and the center tap of the transistors $\mathrm{Q}_{3}$ and $\mathrm{Q}_{4}$; it is used to separate the operating modes of the full-bridge and half-bridge inverters. The secondary components of the matching transformer (inductor $L_{e q}$, resistor $R_{e q}$, and resonant capacitor $C_{r}$ ) are reflected to the primary side and represented as $R, L$, and $C$, respectively.

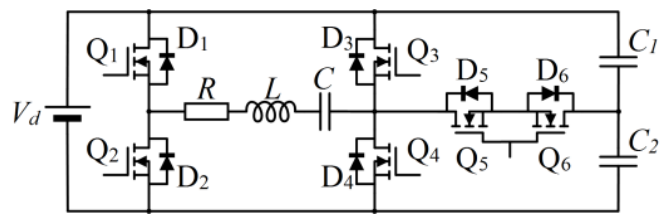

Figure 2. Inverter with the extended topology and the secondary components of the matching transformer reflected to the primary side

The eight topological stages of the extended PDM-SRI are shown in Figure 3. With these stages, four modes of operation for the extended PDM-SRI can be obtained: Mode 1-stages 1 and 2 are used to produce the injection interval $\left(T_{A}\right)$ during which the inverter acts as a square-wave ac-voltage source with the amplitude $V_{d}$ for $m$ cycles of the period $T_{O}$ of the SRI output voltage $v_{O}$; Mode 2-stages 3 and 4 are used to produce the injection interval $\left(T_{C}\right)$ during which the inverter acts as a square-wave ac-voltage source with the amplitude $V_{d} / 2$ for $l$ cycles of $T_{O}$; Mode 3-stages 5 and/or 6 are used to produce the free-wheeling interval $\left(T_{B}\right)$ during which the inverter acts as a zero-voltage source for $n$ cycles of $T_{O}$; Mode 4-stages 7 and 8 are used to produce the rejection interval during which the energy of the resonant circuit is transmitted to $V_{d}$ source. 


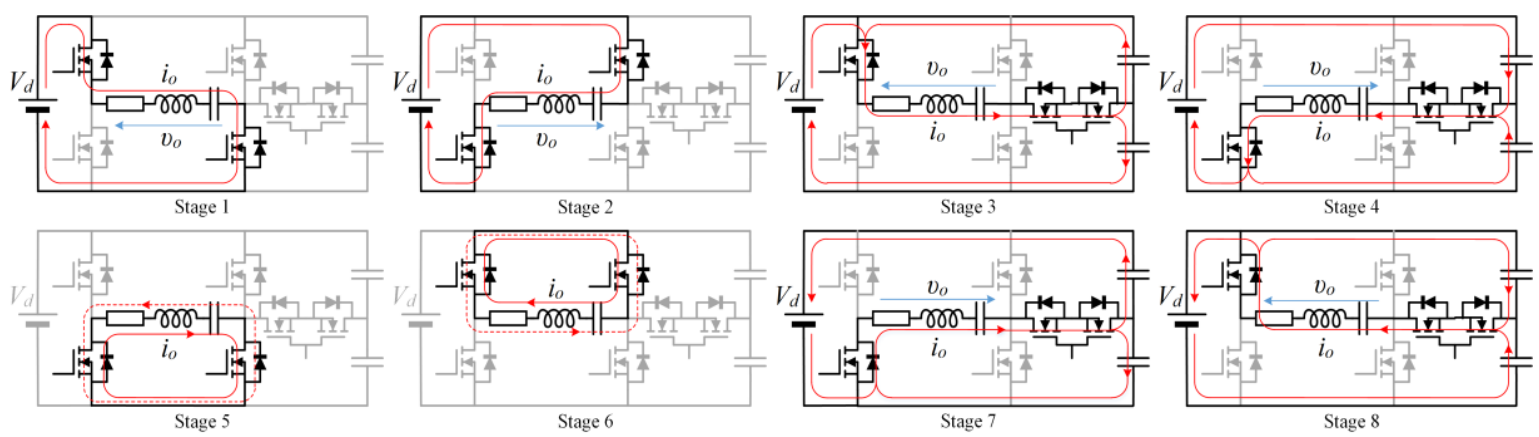

Figure 3. Topological stages of the extended PDM-SRI

To reduce the amplitude fluctuations of $i_{O}$, it is expedient to combine mode 1 with mode 2 , and mode 2 with mode 3 , where mode 2 acts as an intermediate link between modes 1 and 3 . It is not necessary to use mode 4 because when this mode is combined with modes 1 or 2 , the amplitude fluctuations are higher compared to combining mode 3 with modes 1 or 2 . Combining mode 4 with mode 3 is unreasonable; since both stages 5 and 6 ensure a zero-voltage state, it is sufficient to use only one of them to create mode 3 .

Figure 4 shows the PDM control principle for the extended PDM-SRI: case I $-T_{A}$ is combined with $T_{C}$; Case II $-T_{C}$ is combined with $T_{B}$. The duration $T_{M}$ of the PDM sequence can be expressed is being as:

$$
T_{M}=k T_{O}=\left\{\begin{array}{l}
T_{A}+T_{C}=(m+l) T_{O} \quad(\text { in Case } I) \\
T_{C}+T_{B}=(l+n) T_{O}(\text { in Case } I I)
\end{array}\right.
$$

where $k$ is the number of cycles of $T_{O}$ during $T_{M}$. The value of $k$ may be constant for all PDM patterns of the regular and irregular PDM [9], [16], or it can be inconstant [12], [25].

The frequency of PDM sequences is given by:

$$
f_{P D M}=1 / T_{M} \text {. }
$$

For the extended PDM-SRI, the pulse density $D$ of PDM patterns can be expressed is being as:

$$
D=\left\{\begin{array}{lr}
\left(T_{A}+T_{C} / 2\right) / T_{M}=(m+0.5 l) / k & \text { (in Case } I) \\
\left(T_{C} / 2\right) / T_{M}=0.5 l / k & \text { (in Case } I I) .
\end{array}\right.
$$

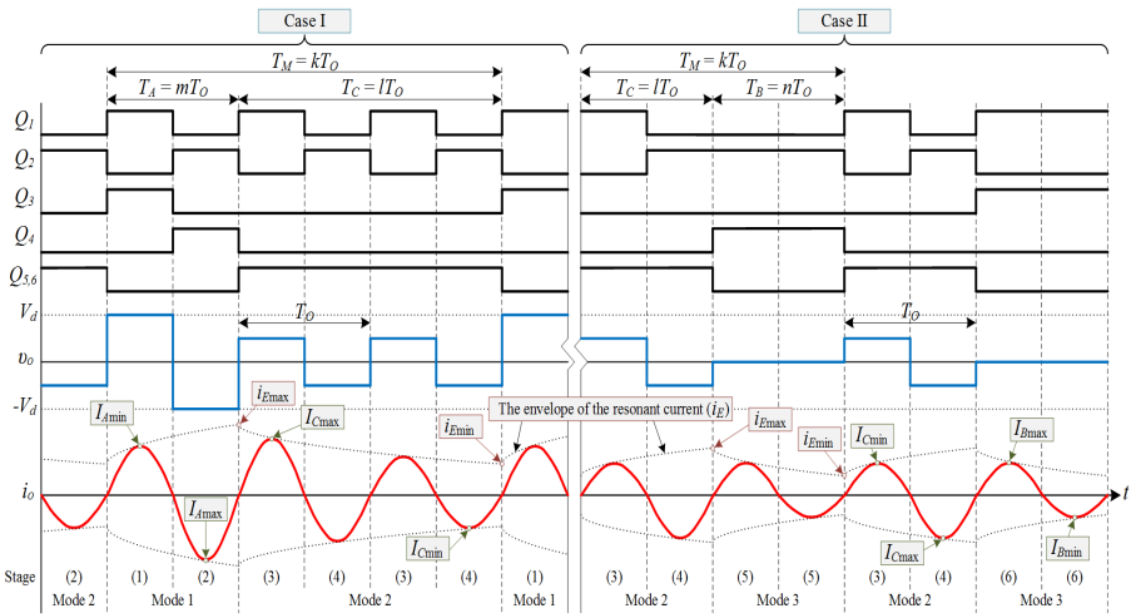

Figure 4. Patterns of control drive sequences and output waveforms in the extended PDM-SRI

\subsection{Analysis of extended pulse-density modulation-series-resonant inverter output current}

Typically, the SRI operates slightly above the resonant frequency to provide ZVS. But to simplify the mathematical analysis, the following two assumptions are made: the SRI operates at the resonant 
frequency $\omega_{O}=1 / \sqrt{L C}$, and the quality factor $Q$ of the resonant circuit is high enough to neglect higher harmonics in $v_{O}$. In this case $v_{O}$ can be represented is being as:

$$
v_{O}(t)=V_{1} \sin \left(\omega_{0} t\right)
$$

where $V_{1}$ is the first harmonic amplitude of $v_{O}\left(V_{1}=4 V_{d} / \pi\right.$ during $T_{A}, V_{1}=2 V_{d} / \pi$ during $T_{C}$, and $V_{1}=0$ during $\left.T_{B}\right)$. In a steady-state operation, when the extended PDM-SRI operates only in mode 1 , the output current $i_{O}$ is given by:

$$
i_{O}(t)=I_{m} \sin \left(\omega_{O} t\right)
$$

where $I_{\mathrm{m}}$ is the maximum current amplitude in the case of $D=1$, and is given by:

$$
I_{m}=\frac{4 V_{d}}{\pi R}
$$

in case I - combination $T_{A}$ and $T_{C}$ Figure 4, the output current $i_{O}$ is given by:

$$
i_{O}(t)= \begin{cases}I_{m} \sin \left(\omega_{O} t\right)\left(1-\frac{1}{2} \frac{1-e^{-\frac{T_{C}}{\tau}}}{1-e^{-\frac{T_{M}}{\tau}}} e^{-\frac{t}{\tau}}\right) & \left(0 \leq t \leq T_{A}\right) \\ I_{m} \sin \left(\omega_{O} t\right)\left(\frac{1}{2}+\frac{1}{2} \frac{1-e^{-\frac{T_{A}}{\tau}}}{1-e^{-\frac{T_{M}}{\tau}}} e^{-\frac{t}{\tau}}\right) & \left(0 \leq t \leq T_{C}\right),\end{cases}
$$

where $\tau$ is the time constant of the envelope of $i_{O}$, and is given by:

$$
\tau=\frac{2 L}{R}=\frac{2 Q}{\omega_{O}}
$$

when the extended PDM-SRI operates only in mode 3 , the output current $i_{O}$ is given by:

$$
i_{O}(t)=\frac{I_{m}}{2} \sin \left(\omega_{O} t\right)
$$

in case II - combination $T_{C}$ and $T_{B}$ Figure 4 , the output current $i_{O}$ is given by:

$$
i_{O}(t)=\left\{\begin{array}{cc}
I_{m} \sin \left(\omega_{O} t\right)\left(\frac{1}{2}-\frac{1}{2} \frac{1-e^{-\frac{T_{B}}{\tau}}}{1-e^{-\frac{T_{M}}{\tau}}} e^{-\frac{t}{\tau}}\right) & \left(0 \leq t \leq T_{C}\right) \\
I_{m} \sin \left(\omega_{O} t\right) \frac{1}{2} \frac{1-e^{-\frac{T_{C}}{\tau}}}{1-e^{-\frac{T_{M}}{\tau}}} e^{-\frac{t}{\tau}} & \left(0 \leq t \leq T_{B}\right) .
\end{array}\right.
$$

It is convenient to submit $\tau$ through the PDM parameters $m, l, n, k$, and the quality factor $Q$. In this way, (7) is given by:

$$
i_{O}(t)= \begin{cases}I_{m} \sin \left(\omega_{O} t\right)\left(1-\frac{1}{2} \frac{1-e^{-\frac{\pi}{Q} l}}{1-e^{-\frac{\pi}{Q} k}} e^{-\frac{\pi}{Q T_{O}} t}\right) & \left(0 \leq t \leq T_{A}\right) \\ I_{m} \sin \left(\omega_{O} t\right)\left(\frac{1}{2}+\frac{1}{2} \frac{1-e^{-\frac{\pi}{Q} m}}{1-e^{-\frac{\pi}{Q} k}} e^{-\frac{\pi}{Q T_{O}} t}\right) & \left(0 \leq t \leq T_{C}\right),\end{cases}
$$

and (10) is given by:

$$
i_{O}(t)=\left\{\begin{array}{cc}
I_{m} \sin \left(\omega_{O} t\right)\left(\frac{1}{2}-\frac{1}{2} \frac{1-e^{-\frac{\pi}{Q} n}}{1-e^{-\frac{\pi}{Q} k}} e^{-\frac{\pi}{Q T_{O}} t}\right) & \left(0 \leq t \leq T_{C}\right) \\
I_{m} \sin \left(\omega_{O} t\right) \frac{1}{2} \frac{1-e^{-\frac{\pi}{Q} l}}{1-e^{-\frac{\pi}{Q} k}} e^{-\frac{\pi}{Q T_{O}} t} & \left(0 \leq t \leq T_{B}\right) .
\end{array}\right.
$$

2.4. Analysis of extended pulse-density modulation-series-resonant inverter output power The average output power is given by [1], [9]: 


$$
P=\frac{1}{T_{M}} \int_{0}^{T_{M}}\left(v_{O} i_{O}\right) d t=\frac{1}{T_{M}} \int_{0}^{T_{M}}\left(V_{1} \sin \left(\omega_{O} t\right) i_{E}(t) \sin \left(\omega_{O} t-\varphi\right)\right) d t
$$

where $\varphi$ is the phase-shift between the fundamental frequencies of $v_{O}$ and $i_{O}$, and $i_{E}(t)$ is the envelope of the resonant current; in case $\mathrm{I} i_{E}(t)$ is given by:

$$
i_{E}(t)= \begin{cases}I_{m}\left(1-\frac{1}{2} \frac{1-e^{-\frac{\pi}{Q} l}}{1-e^{-\frac{\pi}{Q} k}} e^{-\frac{\pi}{Q T_{O}} t}\right) & \left(0 \leq t \leq T_{A}\right) \\ I_{m}\left(\frac{1}{2}+\frac{1}{2} \frac{1-e^{-\frac{\pi}{Q} m}}{1-e^{-\frac{\pi}{Q} k}} e^{-\frac{\pi}{Q T_{O}} t}\right) & \left(0 \leq t \leq T_{C}\right),\end{cases}
$$

and in case II it is given by:

$$
i_{E}(t)=\left\{\begin{array}{cc}
I_{m}\left(\frac{1}{2}-\frac{1}{2} \frac{1-e^{-\frac{\pi}{Q} n}}{\left.1-e^{-\frac{\pi}{Q} k} e^{-\frac{\pi}{Q T_{O}} t}\right)}\right. & \left(0 \leq t \leq T_{C}\right) \\
I_{m} \frac{1}{2} \frac{1-e^{-\frac{\pi}{Q} l}}{1-e^{-\frac{\pi}{Q} k}} e^{-\frac{\pi}{Q T_{O}} t} & \left(0 \leq t \leq T_{B}\right) .
\end{array}\right.
$$

If $\tau \square 1 / \omega_{O},(13)$ changes into the following equation:

$$
P=\frac{2 V_{1}}{\pi} \cos (\varphi) \frac{1}{T_{M}} \int_{0}^{T_{M}} i_{E}(t) d t
$$

The average output power can be calculated through the PDM parameters and $Q$ is being as:

- in the case of full power $(D=1)$, when the SRI operates as a conventional full-bridge SRI

$$
P=P_{\max }=\frac{2}{\pi} V_{d} I_{\mathrm{m}} \cos (\varphi) ;
$$

- in case I

$$
P=P_{\max } \frac{k-0.5 l}{k} ;
$$

- in the case of $D=0.5$, when the SRI operates as a conventional half-bridge SRI

$$
P=\frac{1}{\pi} V_{d} I_{m} \cos (\varphi)
$$

- in case II

$$
P=\frac{P_{\max }}{2}\left[\frac{l}{k}+\frac{Q}{\pi k} \frac{1-e^{-\frac{\pi}{Q} n}}{1-e^{-\frac{\pi}{Q} k}}\left(e^{-\frac{\pi}{Q} l}-1\right)\right] \approx P_{\max } \frac{0.5 l}{k} .
$$

\subsection{Amplitude fluctuation}

The fluctuation of $i_{O}$ can be estimated in two ways: by the peak-to-peak value of the envelope $i_{E}$ of $i_{O}$ [1], and by the absolute peak-to-peak value of the amplitude of $i_{O}$ [29]. It is convenient to estimate the fluctuation by the peak-to-peak value of $i_{E}$ since there are only one maximal value $i_{E \max }$ and one minimal value $i_{E \min }$ of $i_{E}$ within $T_{M}$ Figure 4 . Thus, the fluctuation can be estimated as the difference between $i_{E \max }$ and $i_{E \min }[1]$. The normalized value of the fluctuation by $i_{E}$ is given by:

$$
\Delta I_{E}^{*}=\frac{i_{E \max }-i_{E \min }}{I_{\mathrm{m}}}=\left\{\begin{array}{l}
\frac{1}{2} \frac{1-e^{-\frac{\pi}{Q} m}-e^{-\frac{\pi}{Q} l}+e^{-\frac{\pi}{Q} k}}{1-e^{-\frac{\pi}{Q} k}} \text { (in Case I) } \\
\frac{1}{2} \frac{1-e^{-\frac{\pi}{Q} l}-e^{-\frac{\pi}{Q} n}+e^{-\frac{\pi}{Q} k}}{1-e^{-\frac{\pi}{Q} k}} \text { (in Case II). }
\end{array}\right.
$$


The disadvantage of this approach is that the real absolute peak-to-peak value of the amplitude fluctuation is somewhat less. That is due to the fact that the maximal and minimal amplitudes of $i_{O}$ are at points that differ from the points of $i_{E \max }$ and $i_{E \min }$ Figure 4 . Let's consider the estimation of the fluctuation by the absolute peak-to-peak value of the amplitude of $i_{O}$. The maximal and minimal values of the current amplitude can be found for both active modes of the extended PDM-SRI Figure 4. Assuming that the exponential part of $i_{O}$ does not significantly affect the sinusoidal one during one half-period of $T_{O}$, the minimum amplitude value $I_{\min }$ and the maximum amplitude value $I_{\max }$ of $i_{O}$ can be determined for case $I$ from (11) and for case II from (12) at points corresponding to $0.25 T_{O}$ after the beginning and until the end of the active modes. The normalized minimum and maximum current amplitudes are given by:

- in case I

$$
\begin{aligned}
& I_{A \max }^{*}=\left|\frac{i_{O}\left(T_{A}-0.25 T_{O}\right)}{I_{\mathrm{m}}}\right|=1-\frac{1}{2} \frac{e^{-\frac{\pi}{Q} m}-e^{-\frac{\pi}{Q} k}}{1-e^{-\frac{\pi}{Q} k}} e^{\frac{\pi}{4 Q}}, \\
& I_{A \min }^{*}=\left|\frac{i_{O}\left(0.25 T_{O}\right)}{I_{\mathrm{m}}}\right|=1-\frac{1}{2} \frac{1-e^{-\frac{\pi}{Q} l}}{1-e^{-\frac{\pi}{Q} k} e^{-\frac{\pi}{4 Q}},} \\
& I_{C \max }^{*}=\left|\frac{i_{O}\left(0.25 T_{O}\right)}{I_{\mathrm{m}}}\right|=\frac{1}{2}+\frac{1}{2} \frac{1-e^{-\frac{\pi}{Q} m}}{1-e^{-\frac{\pi}{Q} k} e^{-\frac{\pi}{4 Q}},} \\
& I_{C \text { min }}^{*}=\left|\frac{i_{O}\left(T_{C}-0.25 T_{O}\right)}{I_{\mathrm{m}}}\right|=\frac{1}{2}+\frac{1}{2} \frac{e^{-\frac{\pi}{Q} l}-e^{-\frac{\pi}{Q} k}}{1-e^{-\frac{\pi}{Q} k}} e^{\frac{\pi}{4 Q}} ;
\end{aligned}
$$

- in case II

$$
\begin{aligned}
& I_{C \max }^{*}=\left|\frac{i_{O}\left(T_{C}-0.25 T_{O}\right)}{I_{\mathrm{m}}}\right|=\frac{1}{2}-\frac{1}{2} \frac{e^{-\frac{\pi}{Q} l}-e^{-\frac{\pi}{Q} k}}{1-e^{-\frac{\pi}{Q} k}} e^{\frac{\pi}{4 Q}}, \\
& I_{C \min }^{*}=\left|\frac{i_{O}\left(0.25 T_{O}\right)}{I_{\mathrm{m}}}\right|=\frac{1}{2}-\frac{1}{2} \frac{1-e^{-\frac{\pi}{Q} n}}{1-e^{-\frac{\pi}{Q} k} e^{-\frac{\pi}{4 Q}},} \\
& I_{B \max }^{*}=\left|\frac{i_{O}\left(0.25 T_{O}\right)}{I_{\mathrm{m}}}\right|=\frac{1}{2} \frac{1-e^{-\frac{\pi}{Q} l}}{1-e^{-\frac{\pi}{Q} k} e^{-\frac{\pi}{4 Q}},} \\
& I_{B \min }^{*}=\left|\frac{i_{O}\left(T_{B}-0.25 T_{O}\right)}{I_{\mathrm{m}}}\right|=\frac{1}{2} \frac{e^{-\frac{\pi}{Q} n}-e^{-\frac{\pi}{Q} k}}{2} e^{\frac{\pi}{4 Q}} .
\end{aligned}
$$

In this way, the normalized absolute peak-to-peak current amplitude fluctuation $\Delta I_{\mathrm{m}}^{*}$ is given by:

$$
\Delta I_{\mathrm{m}}^{*}=\left\{\begin{array}{l}
\max \left(I_{A \max }^{*}, I_{C \max }^{*}\right)-\min \left(I_{A \min }^{*}, I_{C \text { min }}^{*}\right)(\text { in Case I) } \\
\max \left(I_{C \text { max }}^{*}, I_{B \text { max }}^{*}\right)-\min \left(I_{C \text { min }}^{*}, I_{B \text { min }}^{*}\right)(\text { in Case II). }
\end{array}\right.
$$

The disadvantage of this approach is that it is necessary to determine which of the maximum amplitudes is higher and which of the minimum ones is lower. Figure 5 shows the relationship between the 
fluctuation and the pulse density $D$, as well as the effectiveness $\eta$ of fluctuations reducing at various values of $Q$, which are obtained for the envelope of current Figure 5(a) and peak-to-peak current Figure 5(b). The effectiveness shows the relative reducing $\Delta I_{E}^{*}$ and $\Delta I^{*}{ }_{\mathrm{m}}$ when using the considered PDM-SRI with the extended topology compared to the PDM-SRI with the full-bridge topology at the same values of $D$, and is given by:

$$
\eta=\frac{\left[\Delta I^{*}(D) \text { 'full-bridgeSRI'] }-\left[\Delta I^{*}(D)\right. \text { 'extended SRI'] }\right.}{\left[\Delta I^{*}(D)\right. \text { 'full-bridgeSRI'] }} 100 \%
$$

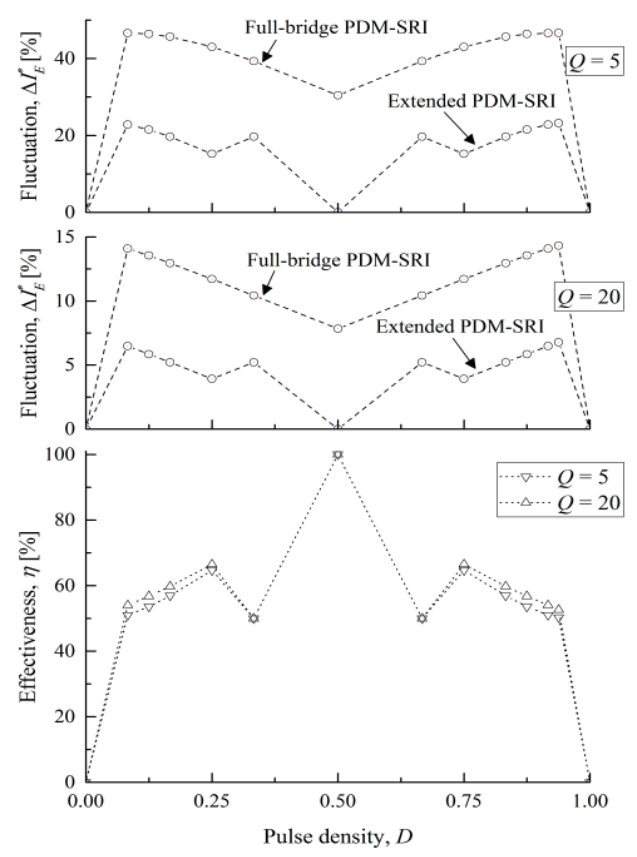

(a)
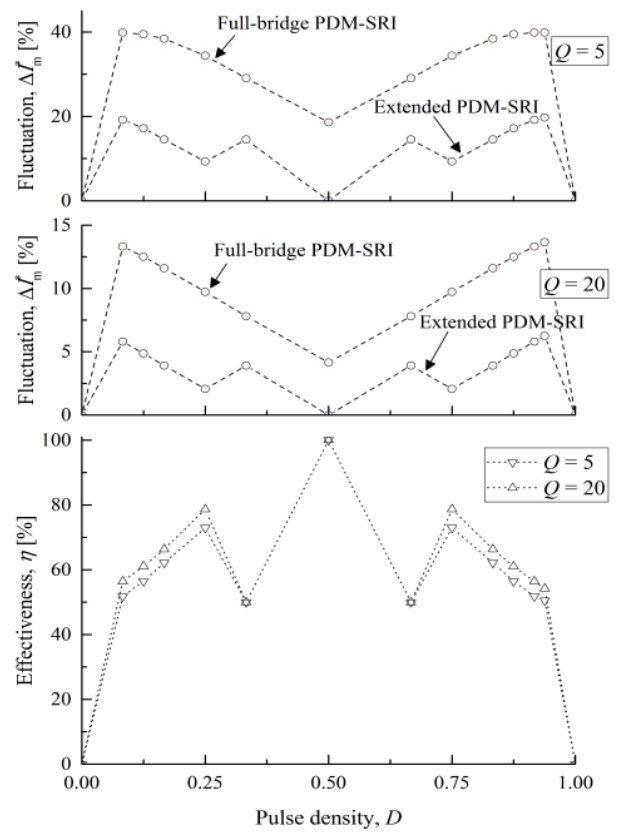

(b)

Figure 5. Relationships comparing between the fluctuation and the effectiveness as functions of $D$ for different values of $Q$ : (a) by envelope of current; (b) by peak-to-peak current

The effectiveness $\eta$ exceeds $50 \%$ and reaches $100 \%$ at $D=0.5$, as shown in Figure 5. The combinations of the PDM parameters used to calculate $\Delta I^{*}$ are given in Table 1 . For the sake of simplicity, the PDM patterns were chosen with inconstant values of $k$, since otherwise PDM patterns with the irregular PDM and constant values of $k$ may contain different values of the PDM parameters within one $T_{M}$, which complicates the determination of $\Delta I^{*}$.

Table 1. Values of PDM parameters

\begin{tabular}{cccccccc}
\hline \multicolumn{3}{c}{ Full-bridge topology } & \multicolumn{5}{c}{ Extended topology } \\
$m$ & $n$ & $k$ & $D$ & $m$ & $l$ & $n$ & $k$ \\
\hline 16 & - & 16 & $1^{\text {a }}$ & 8 & - & - & 8 \\
15 & 1 & 16 & 0.94 & 7 & 1 & - & 8 \\
11 & 1 & 12 & 0.92 & 5 & 1 & - & 6 \\
7 & 1 & 8 & 0.875 & 3 & 1 & - & 4 \\
5 & 1 & 6 & 0.83 & 2 & 1 & - & 3 \\
3 & 1 & 4 & 0.75 & 1 & 1 & - & 2 \\
2 & 1 & 3 & 0.67 & 1 & 2 & - & 3 \\
1 & 1 & 2 & $0.5^{\mathrm{a}}$ & - & 1 & - & 1 \\
1 & 2 & 3 & 0.33 & - & 2 & 1 & 3 \\
1 & 3 & 4 & 0.25 & - & 1 & 1 & 2 \\
1 & 5 & 6 & 0.17 & - & 1 & 2 & 3 \\
1 & 7 & 8 & 0.125 & - & 1 & 3 & 4 \\
1 & 11 & 12 & 0.08 & - & 1 & 5 & 6 \\
- & 16 & 16 & $0^{\mathrm{a}}$ & - & - & 8 & 8 \\
\hline
\end{tabular}

${ }^{\text {a }}$ For these $D$ (except for $D=0.5$ for the full-bridge topology) $v_{O}$ does not have two intervals, and the amplitude does not fluctuate. Thus, $\Delta I_{m}^{*}$ is considered equal 0 , and Imin equal to the amplitude of $i_{O}$ 
When the PDM control method is used, the current control is limited by the available combinations of the PDM parameters. The control system monitors the change in the feedback signal between $i_{O}$ and the level of the current task signal, and being based on the feedback level, selects the appropriate combination of the PDM parameters to provide the required value of $D$. However, when the required value of $D$ cannot be realized with one of the available PDM combination, the control system will change two nearby PDM combinations whose values of $D$ are the closest to the desired one Figure 6. Thus, for $D_{i}<D<D_{i+1}$ (where $D_{i}$ and $D_{i+1}$ are two adjacent values of the pulse density which are the closest to the desired one and can be realized by the available PDM combinations) the amplitude fluctuation level will be determined by the action of the two PDM combinations and can be approximately defined is being as:

$$
\Delta I_{\mathrm{m}}^{*}=I_{\max }^{*}\left(D_{i+1}\right)-I_{\min }^{*}\left(D_{i}\right) .
$$

Figure 7 illustrates similar charts to Figure 5, but for the case when the fluctuation is determined by (32). As can be seen, at a low value of $Q=5$, the effectiveness $\eta$ of fluctuations reducing is more than $40 \%$.

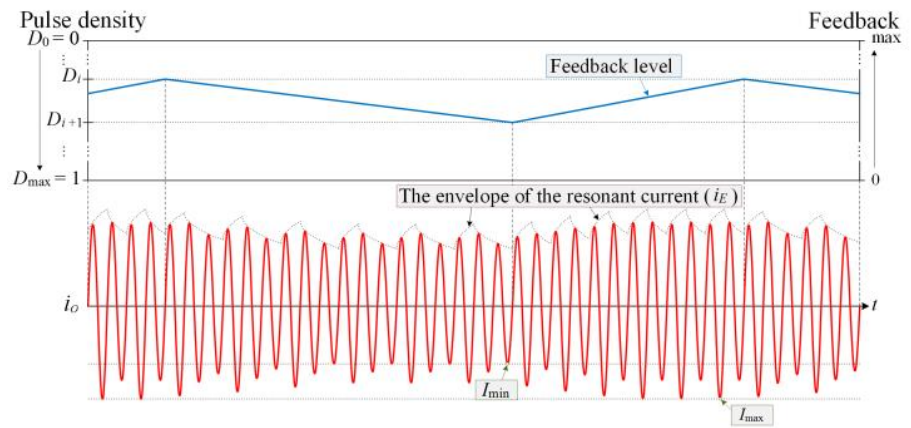

Figure 6. Output current waveform in case of cycling between two nearby combinations of the PDM parameters

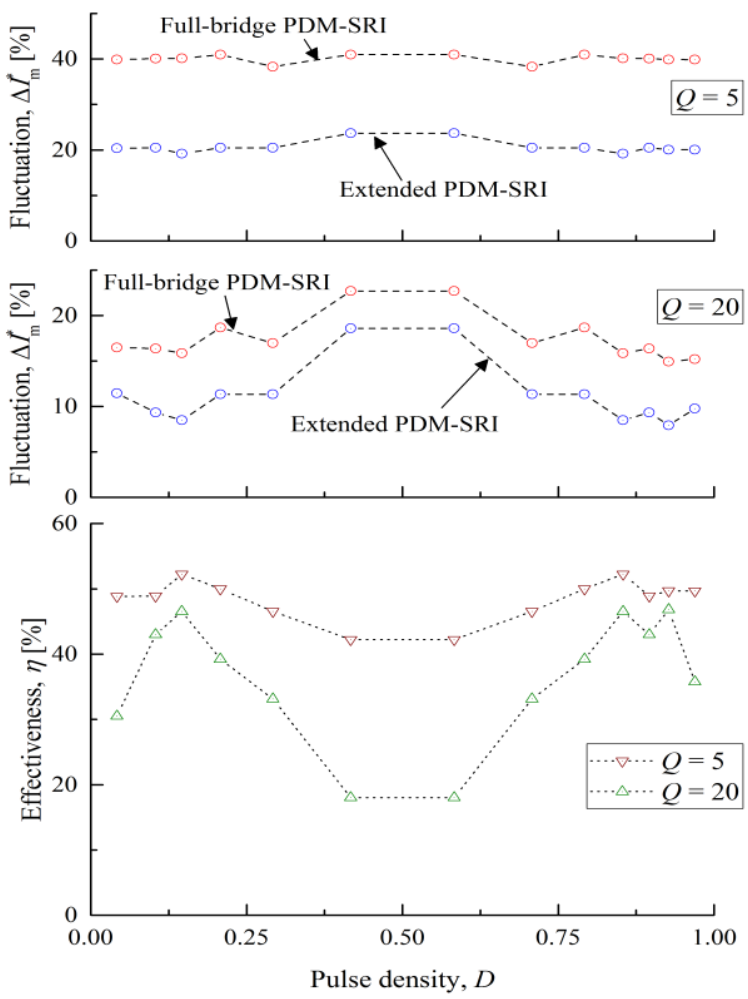

Figure 7. Relationship between the fluctuation and pulse density at $Q$ of 5 and 20, and the effectiveness of fluctuations reducing 


\subsection{Minimal current amplitude}

In order to avoid non-ZVS operations of the SRI transistors, it is necessary to set a certain value of dead-time $T_{D T}$ between the control signals of the SRI transistors. The value of $T_{D T}$ depends, amongst other things, on the current amplitude value [7]-[9]. Thus, on the one hand, $T_{D T}$ can be set constant and must be enough to ensure ZVS under different values of the parameters on which it depends. On the other hand, it can be changeable [31]. In the case of a low value of $Q$, dynamically changing the value of $T_{D T}$ is a complex task and requires high-speed mathematical calculation as well as measurements of current and voltage parameters with high accuracy. In the case of the constant $T_{D T}$, the minimal current amplitude is important and the current fluctuation influences this amplitude value.

Let's consider how using the proposed extended PDM-SRI allows increasing the minimal current amplitude compared to the full-bridge SRI with the traditional PDM control method. Figure 8 shows the difference between normalized values of $I^{*}{ }_{\text {min }}$ for the same $D$ and $Q=5$ for the mentioned above SRIs. As can be seen, if the current regulation is limited by values of $D$ in the range of 0.25 to 1 , the minimum amplitude value can be doubled. Thus, it is possible to reduce the value of $T_{D T}$, and as a consequence, the switching losses will be lower. Or, if the dead-time value does not change, the range of $D$ can be extended by $8.5 \%$.

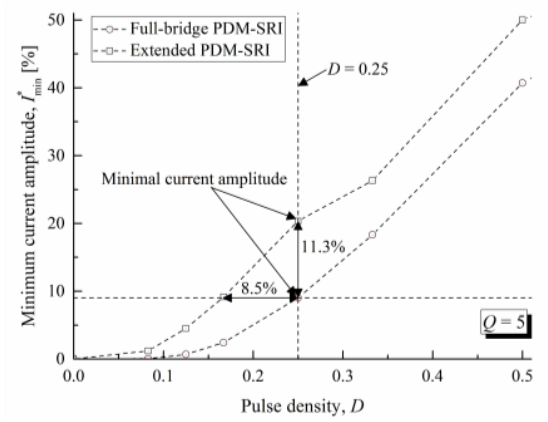

Figure 8. Relationship between the minimal current amplitude and pulse density at $Q=5$

\section{METHOD}

3.1. Drawbacks of extended pulse-density modulation-series-resonant inverter and proposed solutions

Figure 9 shows the SRI with extended topology, where $C_{S}$ is the total capacitance of the snubber capacitor and the parasitic capacitances of the transistors. The main drawback of the extended PDM-SRI presented in [29] is the problem of reverse-recovery of a transistor body-diode. This problem arises in two cases Figure 10: 1) when mode 1 is changed to mode 2, and 2) when mode 3 is changed to mode 2. Let's consider it more deeply (in both cases mentioned, mode 2 is implemented using only stage 5).

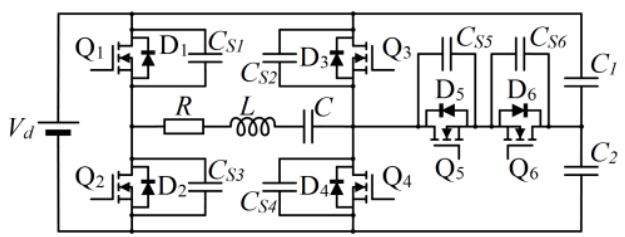

Figure 9. The extended SRI with $C_{S}$

\subsubsection{Reverse recovery problem}

In case I Figure 10 (a), the extended PDM-SRI operates slightly above the resonance frequency. In mode 1 the SRI operates like a conventional full-bridge inverter. There is a certain dead-time $T_{D T}$ between the control signals of the transistors to prevent shoot-through-current. During $T_{D T}, C_{S}$ are fully recharged until the next pair of transistors turns-on. After recharging $C_{S}$, a pair of body-diodes starts to work and the switching of the transistors occurs with ZVS. At the end of mode 1, the bi-directional switch is turned-on within $T_{D T}$. At this moment, one of the active diodes is supplied with half of the inverter input voltage from the capacitor of the half-bride inverter topology, and as a result of this, the shoot-through-current flows through the circuit $\mathrm{D}_{4}-\mathrm{D}_{5}-\mathrm{Q}_{6}$. 
In case II Figure 10 (b), at the end of mode 3 we have a similar situation to the previous one. After recharging $C_{S}$ a pair of the body-diodes works, and the bi-directional switch is turned-on, resulting in a shootthrough-current flowing through the circuit $\mathrm{D}_{4}-\mathrm{D}_{5}-\mathrm{Q}_{6}$. In addition, it is possible to provide that the bidirectional switch would be switched after a current zero-crossing. But in this case, the shoot-through-current will flow through the circuit $\mathrm{D}_{3}-\mathrm{D}_{5}-\mathrm{Q}_{6}$. Therefore, this is not a solution to the problem.

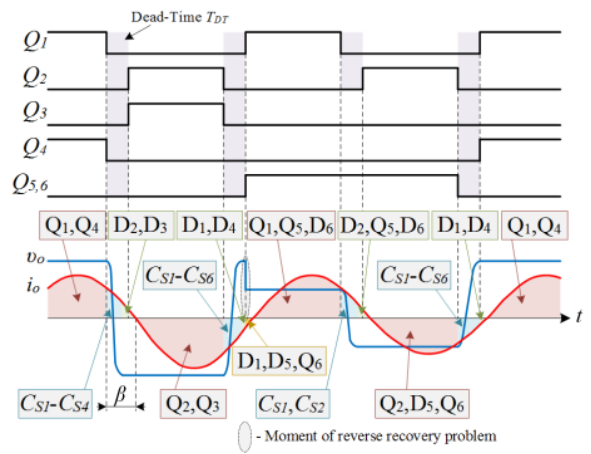

(a)

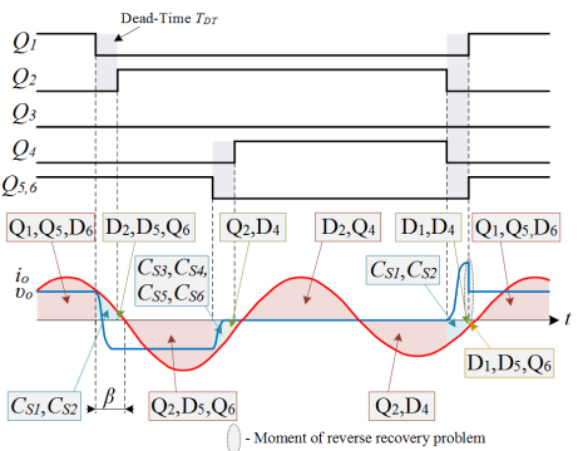

(b)

Figure 10. Switching sequence of the extended PDM-SRI with the diode reverse recovery problem (a) case I and (b) case II

\subsubsection{Solution of reverse recovery problem}

In the described extended topology, the bi-directional switch is implemented with two back-to-back connected transistors $\left(\mathrm{Q}_{5}, \mathrm{Q}_{6}\right)$ in a typical source configuration. A solution to the reverse recovery problem can be found through the change in the control signal of the bi-directional switch. In this way, the control signals $Q_{5}$ and $Q_{6}$ of transistors $\mathrm{Q}_{5}$ and $\mathrm{Q}_{6}$ are different, therefore the bi-directional switch will be seen below as two independent transistors $\mathrm{Q}_{5}$ and $\mathrm{Q}_{6}$. So, Figure 11 (a) shows the switching sequence of the extended PDM-SRI without the diode reverse recovery problem in case I, and Figure 11 (b) for case II, respectively.

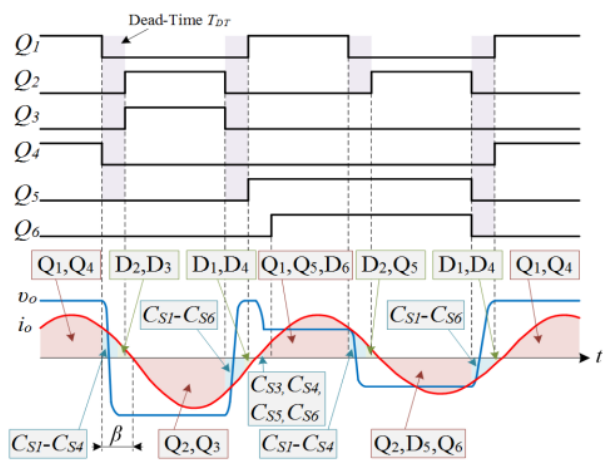

(a)

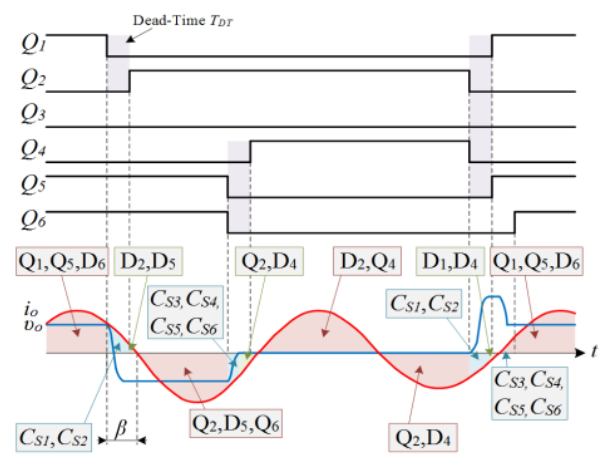

(b)

Figure 11. Switching sequence of the extended PDM-SRI without the diode reverse recovery problem

(a) case I and (b) case II

Both for cases I and II during the diode operation interval (at the end of mode 1 in case I and at the end of mode 3 in case II), it is expedient to turn-on only one of these transistors-the transistor $\mathrm{Q}_{5}$. Since $\mathrm{Q}_{6}$ is still turned-off, there is no problem with the reverse recovery of a transistor body-diode. At the end of the diode operation interval, the current flow changes, and $i_{O}$ flows through $\mathrm{Q}_{1}, \mathrm{Q}_{5}$, and $\mathrm{D}_{6}$. As it is depicted in Figure 11, within this interval, after $C_{S}$ is recharged, $\mathrm{Q}_{6}$ must be turned-on. Such a change in the control signals of $Q_{5}$ and $Q_{6}$ is sufficient to avoid the reverse recovery problem caused by the common control signal $Q_{5,6}$ of the bi-directional switch. 


\subsubsection{Voltage imbalance across capacitors}

The second drawback mentioned in [29] is the voltage imbalance across the capacitors $C_{1}$ and $C_{2}$. This is caused that during mode 2 different currents flow through $C_{1}$ and $C_{2}$, which leads to an increase in voltage across one of the capacitors and a decrease across the other. In case I the envelope of $i_{O}$ falls during mode 2 , causing the voltage across $C_{1}$ to decrease and the voltage across $C_{2}$ to increase. But in case II the envelope of $i_{O}$ rises during mode 2 , causing the opposite situation-the voltage across $C_{l}$ increases, and the voltage across $C_{2}$ decreases. Therefore, it becomes necessary to balance the capacitor voltages.

\subsubsection{Voltage imbalance across capacitors}

A simple solution to this problem is to use the Delon circuit (a bridge voltage doubler circuit), because there is no need to use controlled devices. In this case it is necessary to use two diodes, a transformer and, as an option, a resistor connected as it is shown in Figure 12. The balancing resistor is used as an inrush current limiter and can generally be neglected due to the resistance of the transformer windings of the balancing circuit. Thus, when the voltage across one of the capacitors is higher than the other one, the energy of that capacitor will be transferred to the other one.

In case II during modes 1 and 2, the input voltage of the balancing transformer is half-wave symmetrical due to the pattern sequences of the control signals, and its frequency is equal to the frequency of $v_{o}$. However, when mode 3 is implemented using only stage 5, the input voltage of the balancing transformer will be asymmetrical and the effectiveness of the balancing circuit will be less. Thus, to avoid the asymmetrical voltage, it is reasonable to use mode 3 implemented using stage 5 within one $T_{M}$, and to use mode 3 implemented using stage 6 within the next $T_{M}$. But in this case, when mode 2 is changed to mode 3 , the reverse recovery problem arises, similar to the one when mode 3 is changed to mode 2 , and the shootthrough-current flows through the circuit $\mathrm{Q}_{3}-\mathrm{D}_{4}$. Thus, to avoid this current, $\mathrm{Q}_{3}$ must switch when the direction of $i_{O}$ changes and $\mathrm{D}_{3}$ starts to operate. The resulting sequence of control signals $\mathrm{Q}_{1}-\mathrm{Q}_{4}$ ensuring the ZVS operation of the extended PDM-SRI transistors in case II is shown in Figure 13. In such a way, in case II there is no reverse recovery problem, the input voltage of the balancing transformer will be symmetrical, and its period will be $(2 k-1)$ times more than the period $T_{O}$.

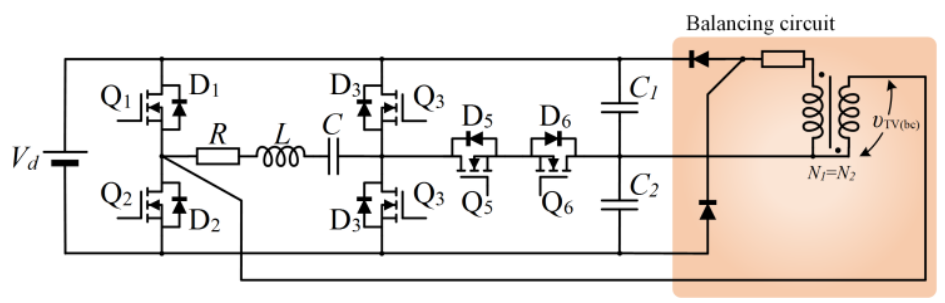

Figure 12. Delon circuit connection

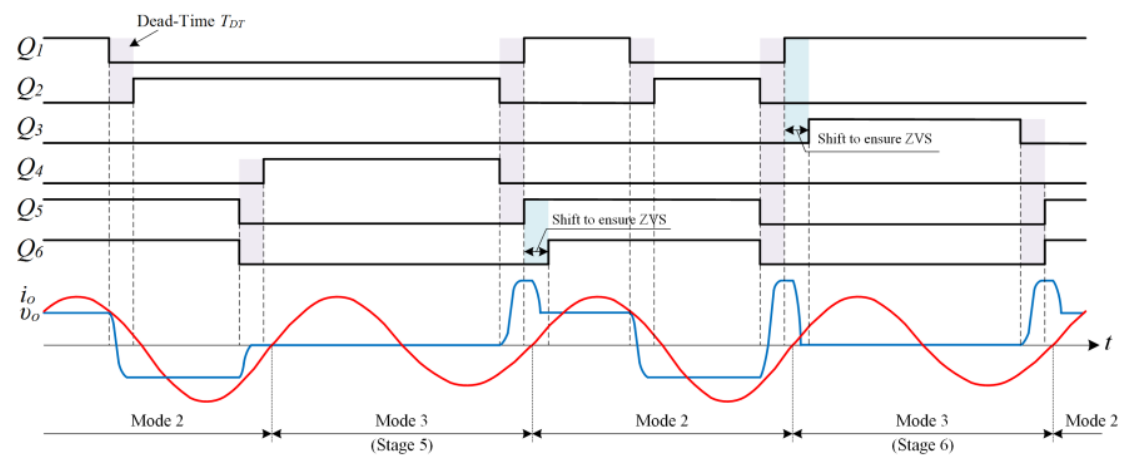

Figure 13. Resulting sequences of control signals to ensure the ZVS operation of the extended PDM-SRI transistors in case II

\subsection{Simulation results}

The extended PDM-SRI has been modeled in the MATLAB/Simulink environment. Simulations are carried out for the two mentioned above cases of combining the operation modes of the extended PDM-SRI. Figure 14 shows waveforms of the output voltage $v_{O}$ and output current $i_{O}$, as well as voltages $v_{C l}, v_{C 2}$ across $C_{1}$ and $C_{2}$, the current through $\mathrm{Q}_{4}$, and the current $i_{\mathrm{Q} 5}$, Q6 through the bi-directional switch, when the

A series-resonant inverter with extended topology and pulse-density-modulation ... (Pavlo Herasymenko) 
mentioned in section 5 drawbacks are present: Figure 14 (a) for case I and Figure 14 (b) for case II. It can be seen in Figure 14 (a) that in case I $v_{C l}$ decreases, and $v_{C 2}$ increases during the modeling process, but in case II there is the opposite situation, as it is shown in Figure 14 (b). In both case I and case II the shoot-throughcurrent flows through the switch devices $\mathrm{Q}_{4}-\mathrm{Q}_{6}$. Figure 15 shows waveforms for the case when the control signals were changed in accordance with the ones proposed in section 5 , and the mentioned above balancing circuit was used Figure 15 (a) for case I and Figure 15 (b) for case II. It can be seen that these changes in the control signals are sufficient to ensure the ZVS operation of the SRI transistors and to avoid the shootthrough-current. In general, there is no imbalance of the voltages $v_{C l}$ and $v_{C 2}$ both in case I and in case II, and $v_{C l}$ and $v_{C 2}$ fluctuate only slightly owing to $C_{1}$ and $C_{2}$ charging and recharging.



(a)


(b)

Figure 14. Waveforms obtained as a result of the simulation when the mentioned drawbacks are present (a) case I and (b) case II

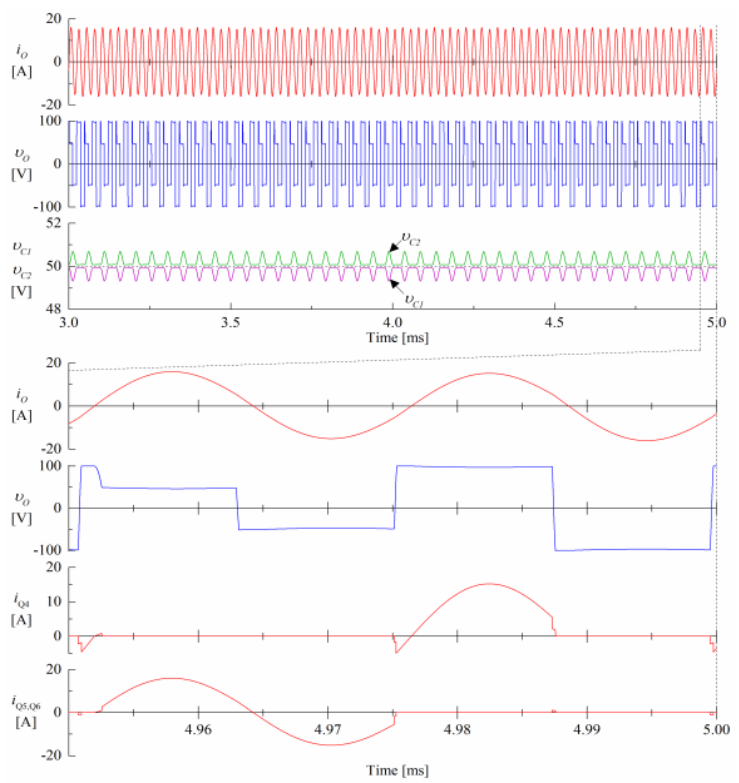

(a)

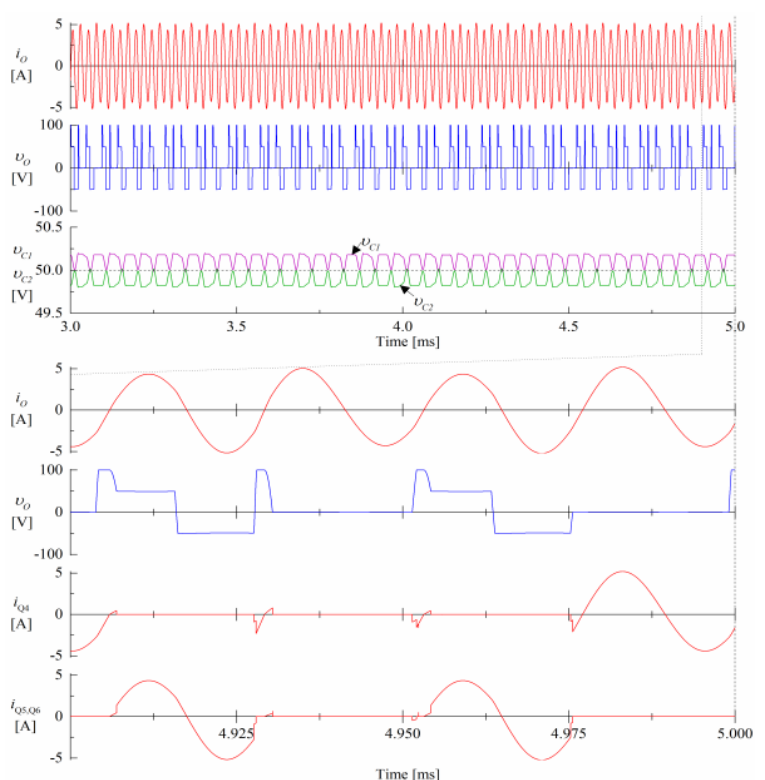

(b)

Figure 15. Waveforms obtained as a result of the simulation when the mentioned drawbacks are absent (a) case I and (b) case II 
Figure 16 shows waveforms of the input voltage $v_{\mathrm{TV}(\mathrm{bc})}$ of the balancing circuit transformer and $v_{0}$ : Figures 16 (a) and (b) when mode 3 is implemented using only stage 5 (under $(k ; l ; n)=(2 ; 1 ; 1)$ Figure 16 (a), and under $(k ; l ; n)=(3 ; 1 ; 2)$ Figures $16(\mathrm{~b})-(\mathrm{d})$ when within one $T_{M}$ mode 2 is combined with implemented using stage 5 mode 3 , and within the next $T_{M}$ mode 2 is combined with implemented using stage 6 mode 3 (under $(k ; l ; n)=(2 ; 1 ; 1)$ Figure $16(\mathrm{c})$, and under $(k ; l ; n)=(3 ; 1 ; 2)$ Figure 16(d). Thus, when using only one of stages 5 or 6 in case II, $v_{\mathrm{TV}(\mathrm{bc})}$ has an asymmetrical duty-cycle. Therefore, the use of the balancing circuit is less effective for balancing $v_{C l}$ and $v_{C 2}$. Besides, $v_{\mathrm{TV}(\mathrm{bc})}$ contains a DC component. So, the alternation of stages 5 and 6 in the way described above makes it possible to ensure the symmetrical $v_{\mathrm{TV}(\mathrm{bc})}$ and, as a result, to increase the effectiveness of balancing $v_{C l}$ and $v_{C 2}$ with the balancing circuit. However, owing to the shape of $v_{\mathrm{TV}(\mathrm{bc})}$ in case II, the balancing circuit transformer must be rated for a frequency with period $(2 k-1)$ times more than $T_{O}$, as is shown in Figure $16(\mathrm{~d})$.

Figure 17 shows waveforms of $v_{C l}$ and $v_{C 2}$, the currents $i_{\mathrm{D}(b c)}$ through the diodes of the balancing circuit, and the currents $\left(i_{C l}\right.$ and $\left.i_{C 2}\right)$ through $C_{1}$ and $C_{2}$, as well as the interrelationship between these voltages and currents. In case I, only one diode of the balancing circuit diodes works; in case II, mode 2another one. The resistor of the balancing circuit together with the resistance of the balancing circuiting transformer windings affects the imbalance of $v_{C l}$ and $v_{C 2}$. On the one hand, the lower the total value of the resistances is, the more effective the balance circuit is. But the inrush current of the balancing circuit operating diode is higher. On the other hand, an increase in the total value of the resistances makes it possible to reduce the inrush current. Still, this current limitation can lead to some imbalance in $v_{C l}$ and $v_{C 2}$ owing to transferred charge and, as a consequence, to the appearance of a DC component in $v_{O}$. In general, the simulation results confirmed the effectiveness of the proposed solutions to eliminate the above-mentioned drawbacks of the extended PDM-SRI.

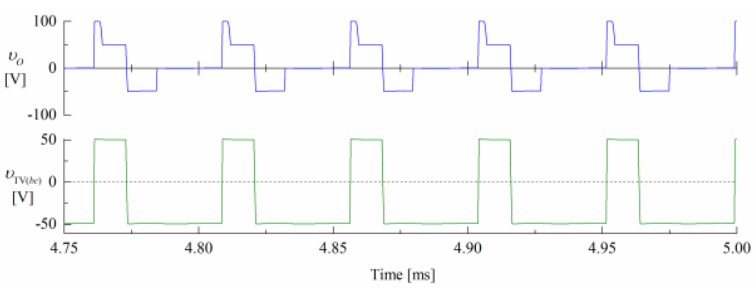

(a)

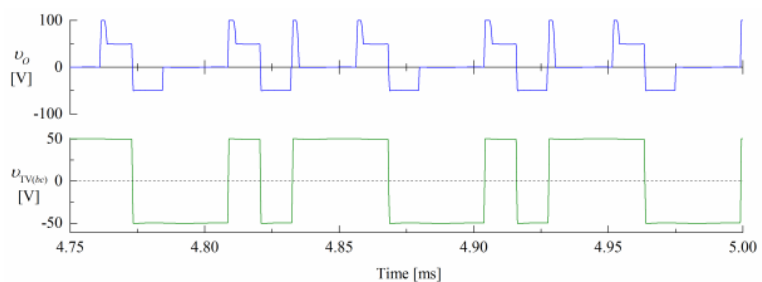

(c)

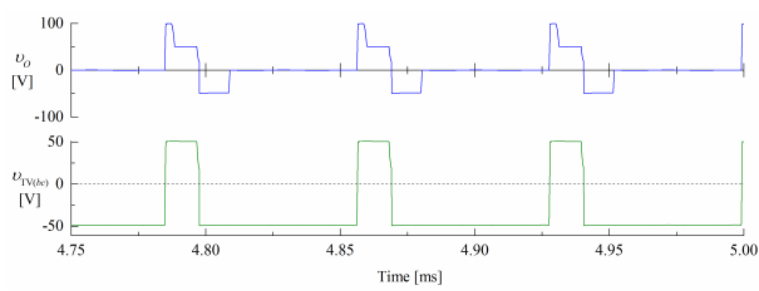

(b)

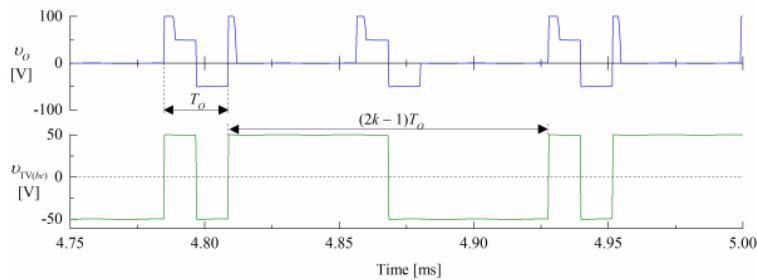

(d)

Figure 16. Waveforms of inverter output voltage and blocking transformer input voltage (a) when using only stage 5 and $(k ; l ; n)=(2 ; 1 ; 1)$, (b) when using only stage 5 and $(k ; l ; n)=(3 ; 1 ; 2)$, (c) when stage 5 and 6 are alternated and $(k ; l ; n)=(2 ; 1 ; 1)$, and $(\mathrm{d})$ when stage 5 and 6 are alternated and $(k ; l ; n)=(3 ; 1 ; 2)$



Figure 17. Waveforms of voltages and currents in case I when $(k ; m ; l)=(2 ; 1 ; 1)$ 


\section{RESULTS AND DISCUSSION}

\subsection{Experimental setup}

The proposed PDM-SRI with the extended topology was verified using an experimental setup with a rated power of $2.2 \mathrm{~kW}$ Figure 18. The structure of the experimental setup is consistent with Figure 1. Discrete $650 \mathrm{~V}, 21 \mathrm{~A} \mathrm{SiC} \mathrm{MOSFETs} \mathrm{(SCT3120ALGC11)} \mathrm{in} \mathrm{the} \mathrm{TO-247-3} \mathrm{package} \mathrm{were} \mathrm{chosen} \mathrm{as}$ switching devices of the extended PDM-SRI.

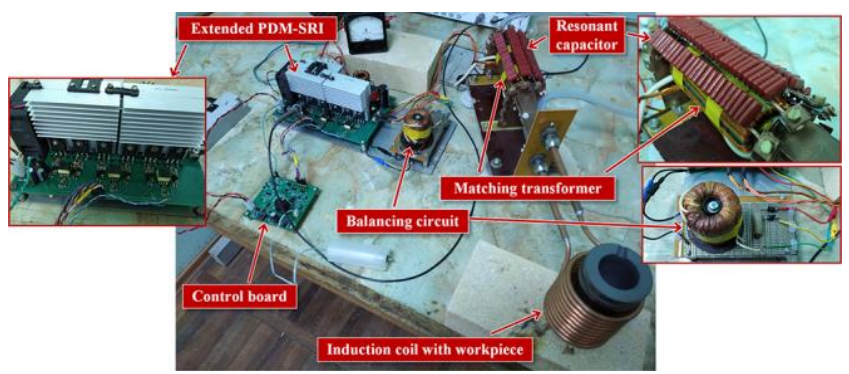

Figure 18. The experimental $2.2 \mathrm{~kW}$ setup

Two main tasks of the experimental setup control system are the generation of the above-described switching sequences and phase synchronization between $v_{O}$ and $i_{O}$ for performing ZVS during the SRI operating. Therefore, the STM32G474RCT6 microcontroller (MC) of the STM32 G4-series was chosen for its ability to generate up to ten PWM signals by its high-resolution timer (HRTIM) [34], [35]. A schematic depiction of the control system structure is shown in Figure 19.

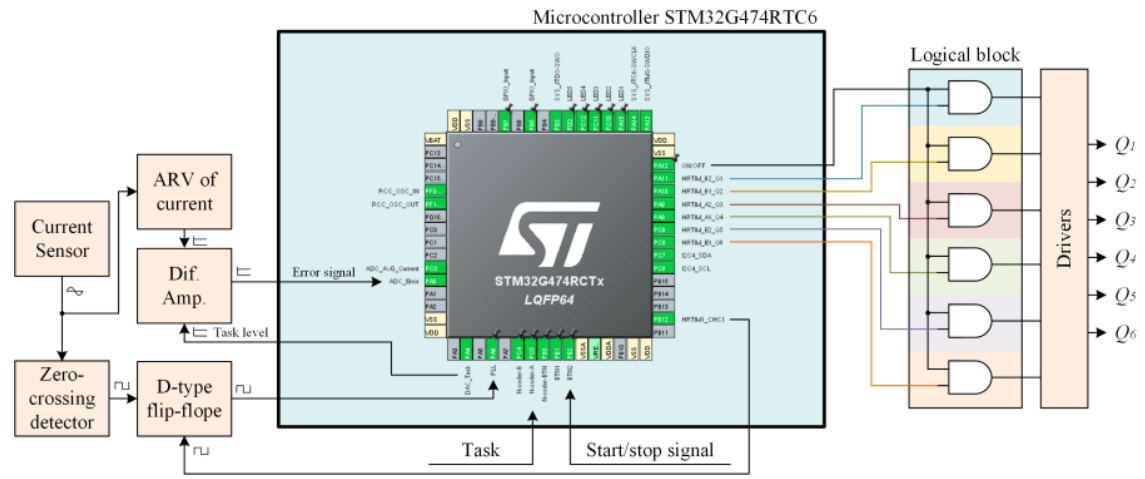

Figure 19. Control system structure based on the microcontroller of the STM32G4 series

Apart from the MC, the control system consists of a current sensor, a current zero-crossing detector, a D-type flip-flop, a logical block, a block of measuring the average rectified value (ARV) of $i_{O}$, an operational amplifier, and drivers of the transistors. During operation, the MC changes the PDM combination according to the error signal produced by the operational amplifier. The output signal of the current sensor is used to determine the zero-crossing and average rectified value of $i_{O}$ by the zero-crossing detector and the block of measuring the ARV, respectively. The phase synchronization is based on a phase-locked-loop (PLL) technique that tracks the phase shift between $v_{O}$ and $i_{O}$. For this, two signals from the zero-crossing detector and the MC are fed to the D-type flip-flop. Based on the signal from the D-type flip-flop, the MC changes the operating frequency. The HRTIM generates six control signals and one shifted signal for phase synchronization. This shifted signal avoids the use of a compensator to eliminate propagation delay between the HRTIM control signals and $v_{O}$. The logical block is used to completely turn off all the SRI transistors of the converter (in case of an inactive state or in case of an emergency).

The HRTIM has to generate six control signals, which differ depending on the operation modes of the extended PDM-SRI and sequencing of changes in the acting PDM combinations. Figure 20 shows the control drive sequences generated by HRTIM for various cases of the PDM combinations. In the presented 
implementation, slave timers A, B, C, E, and the master timer of the HRTIM are used. The master timer handles the frequency control. Thus, the counters of the slave timers are reset on the master period.

To ensure all of the PDM combinations shown in Table 1, HRTIM must generate at least twelve control drive sequence shapes: six in the case of $0.5 \leq D \leq 1$ Figure 20 (a) and six more in the case of $0 \leq D \leq$ 0.5 Figure 20 (b). A kind of shapes the HRTIM has to generate will depend on the sequence of changing the PDM combinations. Thus, the shapes of the control drive sequence of the same PDM combination may differ.

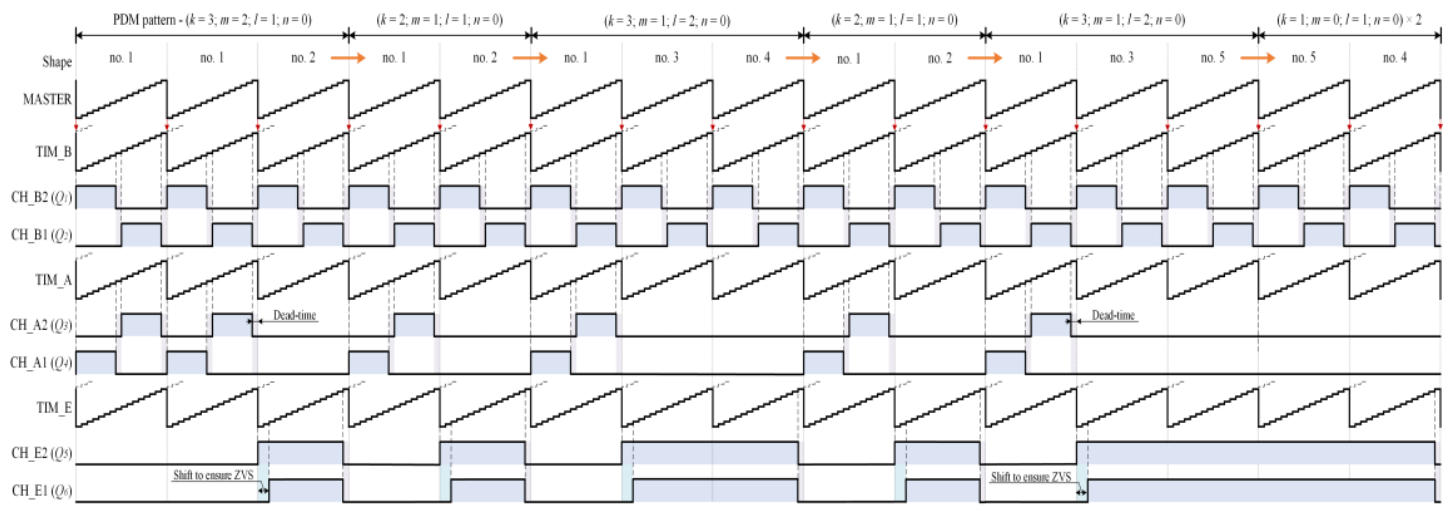

(a)

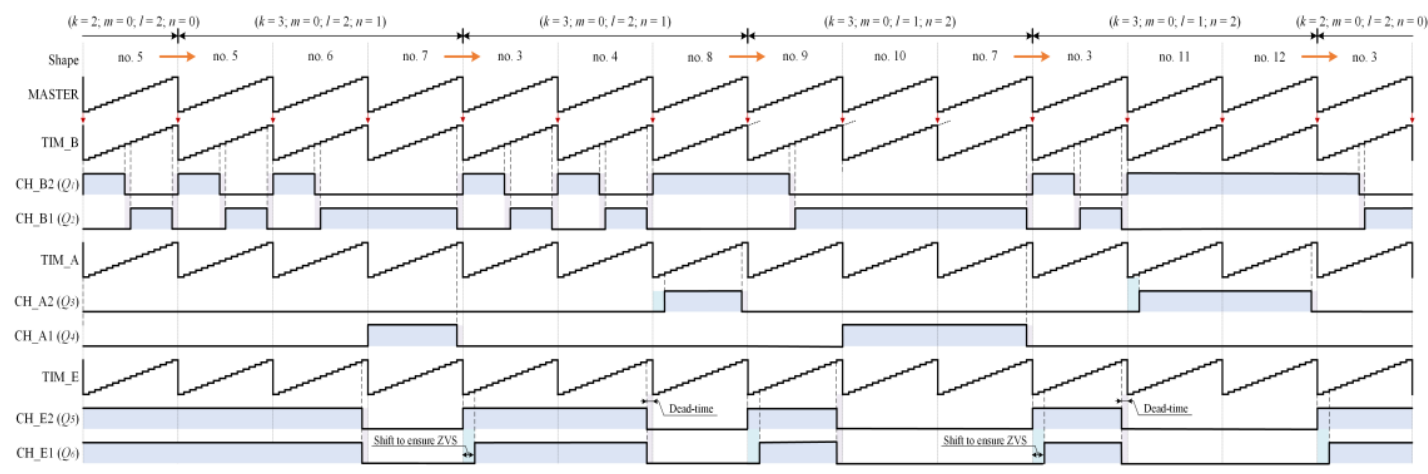

(b)

Figure 20. Shape variants of the control drive sequences (a) in case of $0.5 \leq D \leq 1$ and (b) in case of $0 \leq D<0.5$

\subsection{Experimental waveforms}

Figure 21 shows the experimental $v_{O}$ and $i_{O}$ waveforms of a full-power $(D=1)$ in the steady-state operation obtained by the setup. Experimental waveforms of $v_{O}$ and $i_{O}$ in case of using the extended PDMSRI versus the full-bridge PDM-SRI for different $D$ values are showed in Figure 22 (seen in Appendix). Figures 22 (a)-(c) on the left side show experimental waveforms for the extended PDM-SRI in the case of combining modes 1 and 2, when $D=0.83, D=0.75$, and $D=0.67$, respectively. Figure 22 (d) on the left side shows experimental waveforms in the case of modes 2 , when $D=0.5$ (the SRI operates as a conventional half-bridge inverter). Figure 22 (e) on the left side shows experimental waveforms for the extended PDMSRI in the case of combining modes 2 and 3, when $D=0.33$. On the right side of Figure 22 experimental waveforms for the full-bridge PDM-SRI, respectively for the same $D$ value of the extended PDM-SRI, are shown. The operating frequency of the SRI was close to $34 \mathrm{kHz}$. Figure 23 shows the relationship between the current amplitude fluctuation and the output power when using the proposed PDM-SRI versus the fullbridge SRI with the traditional PDM. The fluctuation was calculated from the waveforms of $i_{O}$. The $Q$ value during the experiments was close to 6 .

The resulting waveforms are fully in line with expectations. Since different shapes are required based on the sequence of changing the PDM combinations, different sequences have been stored in the MC memory for the same pulse density, and MC choses the appropriate sequence being based on the change of $D$. So, there is no problem with changing the sequence. Through ongoing experiments, the $T_{D T}$ value was kept constant. To reduce the switching losses in the inverter transistors, the $T_{D T}$ value must be changeable [31]. Owing to fluctuations, changing the $T_{D T}$ every $T_{O}$ is challenging. On the other hand, the required $T_{D T}$ value can only be determined for the minimal current amplitude. But calculating it correctly and promptly at a low $Q$ value is a complex task. To

A series-resonant inverter with extended topology and pulse-density-modulation ... (Pavlo Herasymenko) 
avoid inrush current during start-up, the initial frequency of the extended PDM-SRI was $50 \mathrm{kHz}$. During the start-up process, it is possible to obtain the non-ZVS operation of the extended PDM-SRI if the time shift in the control signals of $Q_{3}$ and/or $Q_{6}$ is too small, as shown in Figure 24(a). On the other hand, in a wide range of operating frequencies, it is also possible to obtain non-ZVS if the time shift is too large. Therefore, the time shift was set dynamically variable and equal to a quarter of the actual $T_{O}$. For this case, in Figures $24(\mathrm{~b})$ and $24(\mathrm{c})$ the waveforms of $v_{O}$ and $i_{O}$ within the start-up process and in the steady-state operation, respectively, are shown. In both cases, the extended PDM-SRI operates with ZVS.



Figure 21. Experimental waveforms of $v_{O}$ and $i_{O}$ in case of full-power operation $(D=1)$

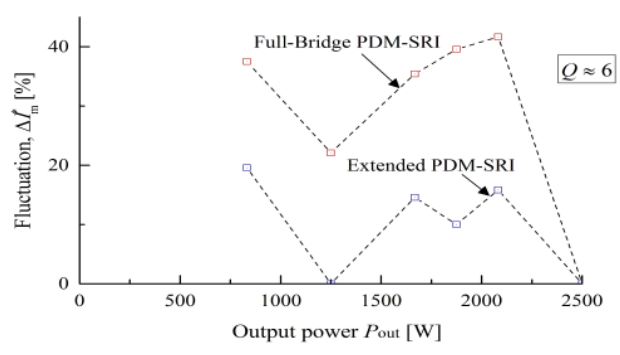

Figure 23. Relationship between the fluctuation and the output power

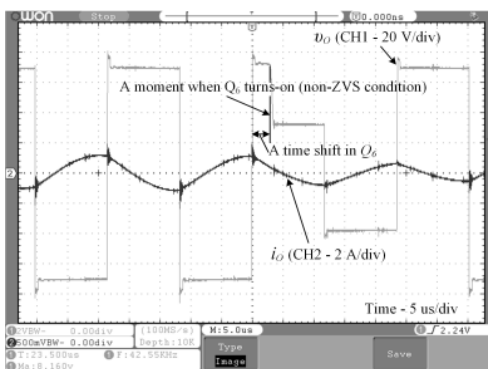

(a)

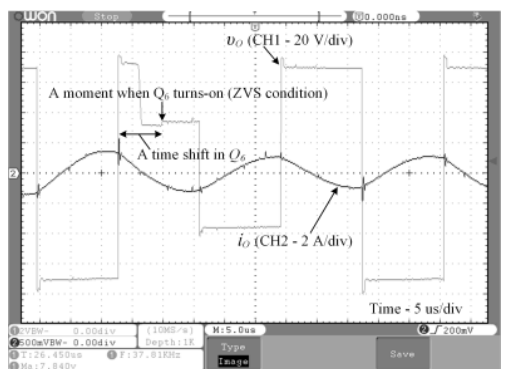

(b)



(c)

Figure 24. Waveforms of $v_{O}$ and $i_{O}$ at a low input voltage of the extended PDM-SRI $(V d=70 \mathrm{~V})(\mathrm{a})$ within start-up process and under non-ZVS condition; constant time shift in $Q_{6} / Q_{3}$, (b) within start-up process and under ZVS condition; dynamically variable time shift in $Q_{6} / Q_{3}$, and (c) in steady-state operation and under ZVS condition; dynamically variable time shift in $Q_{6} / Q_{3}$

In general, the experimental results confirm the feasibility and performance of the proposed extended PDM-SRI. It is also worth noting the significant size of the balancing transformer due to the low frequency of its input voltage at low values of $D$. In the experimental setup, the size was close to half the size of the matching transformer. To reduce the dimension of the balancing transformer, it is possible for $D<0.5$ to use the PFM control method instead of the PDM one. In this case, the input voltage frequency of the balancing transformer will be equal to that of the matching one. Furthermore, one diode of the balancing circuit will be needless. Moreover, there will be no amplitude fluctuations of the SRI current. On the other hand, the dynamic losses in the SRI transistors will be higher.

\section{CONCLUSION}

The extended PDM-SRI for induction heating applications which is proposed and analyzed in the paper, has serious advantages in comparison with other types of PDM-SRIs. Such kind of the extended PDMSRI allows to reduce the fluctuation of the SRI output current compared to the traditional PDM-SRI or reduces the number of required switching devices compared to a modular converter, which is based at least on two inverters connected in series, with an interleaved or a stepped PDM control method. A PDM control method and soft-switching operation of the proposed SRI were theoretically analyzed, explained, and verified with simulation. The experimental setup of a $2.2 \mathrm{~kW}$ extended PDM-SRI has confirmed its feasibility. 


\section{APPENDIX}


(a)
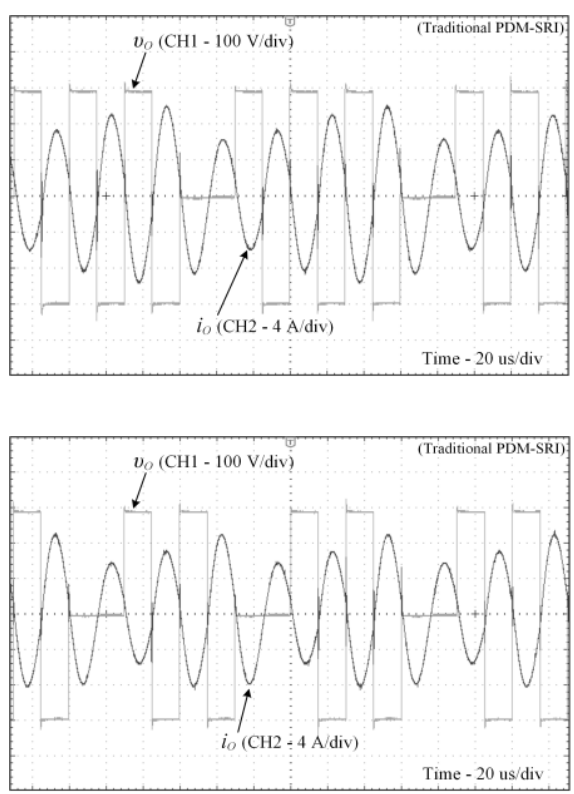

(b)

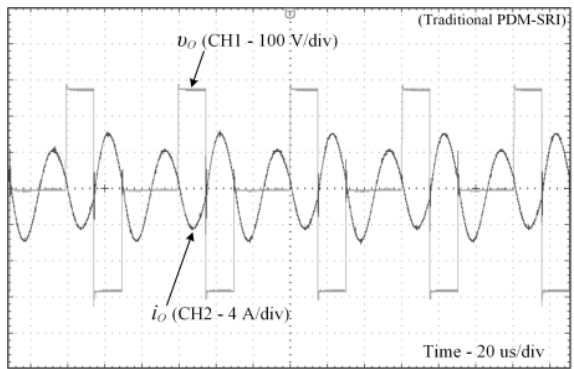

(c)

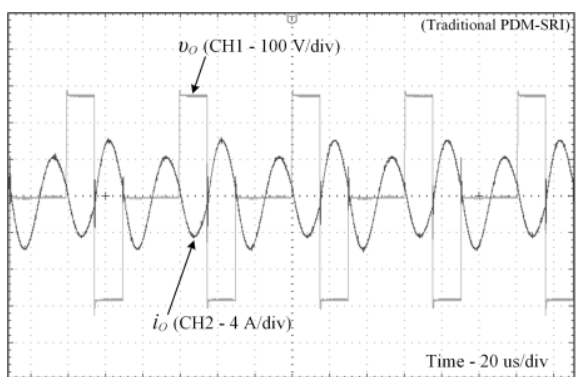

(d)

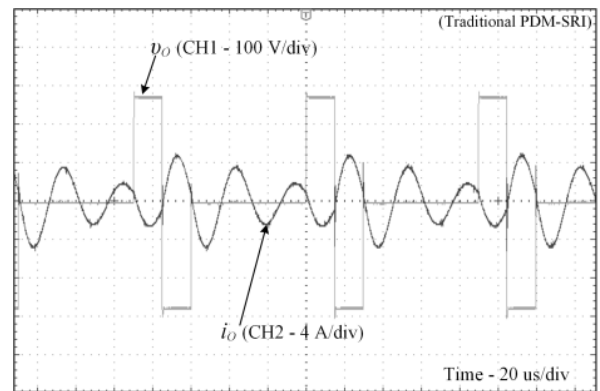

(e)

Figure 22. Experimental waveforms of $v_{O}$ and $i_{O}$ in case of using the extended PDM-SRI versus the full-bridge PDM-SRI (a) in case of $D=0.83$, (b) in case of $D=0.75$, (c) in case of $D=0.67$, (d) in case of $D=0.5$ and (e) in case of $D=0.33$ 


\section{REFERENCES}

[1] V. Esteve, et al., "Enhanced Pulse-Density-Modulated Power Control for High-Frequency Induction Heating Inverters," in IEEE Transactions on Industrial Electronics, vol. 62, no. 11, pp. 6905-6914, Nov. 2015, doi: 10.1109/TIE.2015.2436352.

[2] J. Hu, C. Bi, K. Jia and Y. Xiang, "Power Control of Asymmetrical Frequency Modulation in a Full-Bridge Series Resonant Inverter," in IEEE Transactions on Power Electronics, vol. 30, no. 12, pp. 7051-7059, Dec. 2015, doi: 10.1109/TPEL.2014.2384523.

[3] M. Fan, L. Shi, Z. Yin, L. Jiang, and F. Zhang, "Improved Pulse Density Modulation for Semi-bridgeless Active Rectifier in Inductive Power Transfer System," in IEEE Transactions on Power Electronics, vol. 34, no. 6, pp. 5893-5902, June 2019, doi: 10.1109/TPEL.2018.2867902.

[4] H. Li, J. Fang, S. Chen, K. Wang, and Y. Tang, "Pulse Density Modulation for Maximum Efficiency Point Tracking of Wireless Power Transfer Systems," in IEEE Transactions on Power Electronics, vol. 33, no. 6, pp. 5492-5501, June 2018, doi: 10.1109/TPEL.2017.2737883.

[5] H. Setiadi and H. Fujita, "Light-Load Switching-Loss Elimination Utilizing Pulse Density Modulation for Switched-CapacitorBased Resonant Converters," 2019 IEEE Energy Conversion Congress and Exposition (ECCE), 2019, pp. 4734-4740, doi: 10.1109/ECCE.2019.8912624.

[6] X. Sheng and L. Shi, "An Improved Pulse Density Modulation Strategy Based on Harmonics for ICPT System," in IEEE Transactions on Power Electronics, vol. 35, no. 7, pp. 6810-6819, July 2020, doi: 10.1109/TPEL.2019.2958644.

[7] H. Fujita, H. Akagi, K. Sano, K. Mita, and R. H. Leonard, "Pulse density modulation based power control of a 4 kW 400 kHz voltage-source invertor for induction heating applications," Conference Record of the Power Conversion Conference-Yokohama 1993, 1993, pp. 111-116, doi: 10.1109/PCCON.1993.264238.

[8] V. Esteve, et al., "Improving the Efficiency of IGBT Series-Resonant Inverters Using Pulse Density Modulation," in IEEE Transactions on Industrial Electronics, vol. 58, no. 3, pp. 979-987, March 2011, doi: 10.1109/TIE.2010.2049706.

[9] H. Fujita and H. Akagi, "Pulse-density-modulated power control of a $4 \mathrm{~kW}, 450 \mathrm{kHz}$ voltage-source inverter for induction melting applications," in IEEE Transactions on Industry Applications, vol. 32, no. 2, pp. 279-286, March-April 1996, doi: $10.1109 / 28.491475$

[10] A. Pholsriphim, S. Nurach, and W. Lenwari, "Half-bridge resonance inverter for induction heating using digital-controlled pulse density modulation technique," in 2017 12th IEEE Conference on ICIEA, June 2017, pp. 1084-1086, doi: 10.1109/ICIEA.2017.8283001.

[11] H H. Fujita and H. Akagi, "Control and performance of a pulse-density-modulated series-resonant inverter for corona discharge processes," in IEEE Transactions on Industry Applications, vol. 35, no. 3, pp. 621-627, May-June 1999, doi: 10.1109/28.767013.

[12] T. -. Wu and J. -. Hung, "A PDM controlled series resonant multi-level converter applied for X-ray generators," 30th Annual IEEE Power Electronics Specialists Conference. Record. (Cat. No.99CH36321), 1999, pp. 1177-1182 vol.2, doi: 10.1109/PESC.1999.785661.

[13] A. Karafil, H. Ozbay, and S. Oncu, "Power control of resonant converter MPPT by pulse density modulation," 2017 10th International Conference on Electrical and Electronics Engineering (ELECO), 2017, pp. 360-364.

[14] A. Sandali, A. Cheriti, and P. Sicard, "Comparison of the various PDM control modes," 2004 IEEE International Conference on Industrial Technology, 2004. IEEE ICIT '04., vol. 2, pp. 574-579, 2004, doi: 10.1109/ICIT.2004.1490138.

[15] H. Calleja and J. Pacheco, "Power distribution in pulse-density modulated waveforms," 2000 IEEE 31st Annual Power Electronics Specialists Conference. Conference Proceedings (Cat. No.00CH37018), vol. 3, pp. 1457-1462, 2000, doi: 10.1109/PESC.2000.880522.

[16] A. Karafil, H. Ozbay, and S. Oncu, "Comparison of regular and irregular 32 pulse density modulation patterns for induction heating," IET Power Electronics, vol. 14, no. 1, pp. 10-12, December 2020, doi: 10.1049/pel2.12012.

[17] X. Sheng, L. Shi, and M. Fan, “An Improved Pulse Density Modulation of High-Frequency Inverter in ICPT System," in IEEE Transactions on Industrial Electronics, vol. 68, no. 9, pp. 8017-8027, Sept. 2021, doi: 10.1109/TIE.2020.3013782

[18] P. Y. Herasymenko, "A transistor resonant voltage inverter with pulse density modulation for induction heating equipment," Technical Electrodynamics, no. 6, pp. 24-28, 2015.

[19] Y. Liu and X. He, "PDM and PFM hybrid control of a series-resonant inverter for corona surface treatment," IEE ProceedingsElectric Power Applications, vol. 152, no. 6, pp. 1445-1450, December 2005, doi: 10.1049/ip-epa:20045270.

[20] J. Shen, H. Ma, W. Yan, J. Hui and L. Wu, "PDM and PSM Hybrid Power Control of a Series-Resonant Inverter for Induction Heating Applications," 2006 1ST IEEE Conference on Industrial Electronics and Applications, 2006, pp. 1-6, doi: 10.1109/ICIEA.2006.257060.

[21] A. Namadmalan, "Universal Tuning System for Series-Resonant Induction Heating Applications," in IEEE Transactions on Industrial Electronics, vol. 64, no. 4, pp. 2801-2808, April 2017, doi: 10.1109/TIE.2016.2638399.

[22] P. Herasymenko, "Combined PS-PDM control method for voltage-source series-resonant inverter," Przeglad Elektrotechniczny, vol. 97, no. 5, pp. 40-45, May 2021, doi: 10.15199/48.2021.05.07.

[23] Y. Uesugi, T. Imai, K. Kawada and S. Takamura, "Fundamental and third harmonic operation of SIT inverter and its application to RF thermal plasma generation," Proceedings of the Power Conversion Conference-Osaka 2002 (Cat. No.02TH8579), vol. 3, pp. 1473-1478, 2002, doi: 10.1109/PCC.2002.998191.

[24] M. Swadowski, K. Zygon, and A. Jąderko, "Wysokoczęstotliwościowy przekształtnik z obwodami rezonansowymi pracującymi z wielokrotnością częstotliwości przekształtnika na przykładzie zastosowania w nagrzewnicach indukcyjnych, (in English: Highfrequency converters with resonant circuits working with multiple converter frequency on an example of use in induction heaters)," Przeglad Elektrotechniczny, vol. 94, no. 5, pp. 143-146, May 2018, doi: 10.15199/48.2018.05.25.

[25] P. Herasymenko, "Soft Start-up Output Current of PDM-based Series-Resonant Converter for Induction Heating Application," 2019 IEEE 2nd Ukraine Conference on Electrical and Computer Engineering (UKRCON), 2019, pp. 570-575, doi: 10.1109/UKRCON.2019.8879832.

[26] E. J. Dede, J. Jordán, and V. Esteve, "The practical use of SiC devices in high power, high frequency inverters for industrial induction heating applications," 2016 IEEE 2nd Annual Southern Power Electronics Conference (SPEC), 2016, pp. 1-5, doi: 10.1109/SPEC.2016.7846163.

[27] H. Sarnago, Ó. Lucía, and J. M. Burdío, "Interleaved Resonant Boost Inverter Featuring SiC Module for High-Performance Induction Heating," in IEEE Transactions on Power Electronics, vol. 32, no. 2, pp. 1018-1029, Feb. 2017, doi: 10.1109/TPEL.2016.2554607. 
[28] P. Herasymenko and V. Pavlovskyi, "Soft Start-up Strategy of Pulse-Density-Modulated Series-Resonant Converter for Induction Heating Application," International Journal of Power Electronics and Drive System (IJPEDS), vol. 12, no. 1, pp. 258-272, March 2021, doi: 10.11591/ijpeds.v12.i1.pp258-272.

[29] P. Herasymenko and O. Yurchenko, "An Extended Pulse-Density-Modulated Series-Resonant Inverter for Induction Heating Applications," 2020 IEEE 61th International Scientific Conference on Power and Electrical Engineering of Riga Technical University (RTUCON), 2020, pp. 1-8, doi: 10.1109/RTUCON51174.2020.9316617.

[30] L. Takau, "Improved modelling of induction and transduction heaters: doctoral thesis," Doctoral thesis, University of Canterbury, Christchurch, New Zealand, 2015.

[31] G. M. Segura, "Induction heating converter's design, control and modeling applied to continuous wire," PhD Thesis. PhD thesis, UPC, 2012. 26, 27, 28, 29, 33, 2012.

[32] United States Patent. Patent no. US7888880B2, "Dielectric barrier discharge lamp system and driving method thereof having relatively better performance in startup and re-startup of dimming".

[33] B.-R. Lin, “Analysis of Hybrid Converter with Wide Voltage Range Operation,” Journal of Power Electronics, vol. 19, no. 5, pp. 1099-1107, Sep. 2019, doi: 10.6113/JPE.2019.19.5.1099.

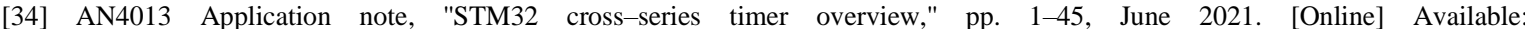
https://www.st.com/content/ccc/resource/technical/document/application_note/54/0f/67/eb/47/34/45/40/DM00042534.pdf/files/D M00042534.pdf/jcr:content/translations/en.DM00042534.pdf.

[35] AN4539 Application note, "HRTIM cookbook," pp. 1-60, January 2020. [Online] Available: https://www.st.com/content/ccc/resource/technical/document/application_note/13/d6/48/9d/11/11/4c/08/DM00121475.pdf/files/D M00121475.pdf/jcr:content/translations/en.DM00121475.pdf.

\section{BIOGRAPHIES OF AUTHORS}
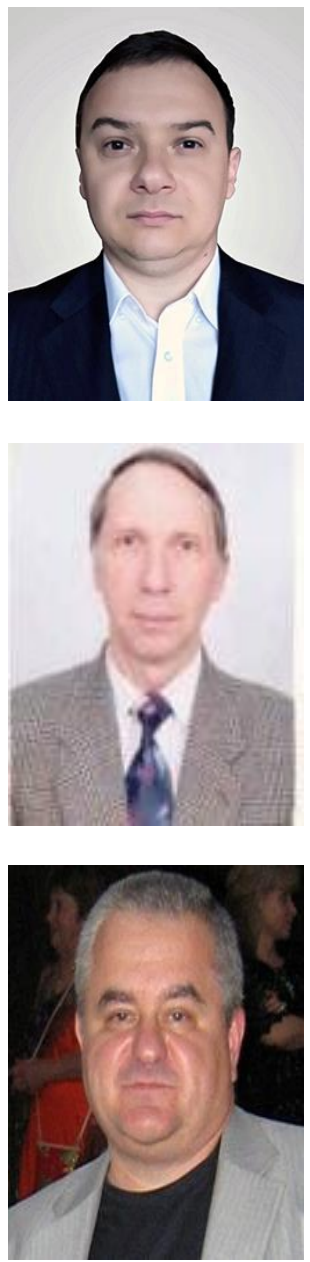

Pavlo Herasymenko (iD) SC P is a senior researcher in the Institute of Electrodynamics, Kyiv, Ukraine. He received the B.S. and M.S. degrees in electrical engineering from the National Technical University of Ukraine "Kyiv Polytechnic Institute", Kyiv, Ukraine, in 2004 and 2006, respectively, and Ph.D. degree from the Institute of Electrodynamics of the National Academy of Sciences of Ukraine, Kyiv, Ukraine, in 2010. His main research interests are the design and application of high-power converters for induction heating equipment, digital control, and custom power devices. He can be contacted at email: herasymenko@ieee.org.

Volodymyr Pavlovskyi (D) 8d SC P was born in Bila Tserkva, Ukraine, on January, 16, 1947. $\mathrm{He}$ received the B.S. and M.S. degrees in radio engineering from the Kyiv Polytechnic Institute at Kyiv, Ukraine, in 1971, and the Ph. D. degree in signal processing in 1980. From 1982, he was a Senior Research Worker with several research institutes, where he studied electromagnetic interferences in power converters. Since 1993, he has been a Senior Research Worker with the Institute of Electrodynamics of the National Academy of Sciences of Ukraine. His current research interests include electromagnetic compatibility problems in power inverters and converters. He can be contacted at email: vopvl.2012@gmail.com.

Oleg Yurchenko (iD SC P is the head of the department of transistor converters of the Institute of Electrodynamics of the National Academy of Sciences of Ukraine. He graduated from Kyiv Polytechnic Institute in 1986. He received the qualification of an electronics engineer in the specialty "Industrial Electronics". He received Ph.D. degree from the Institute of Electrodynamics of the National Academy of Sciences of Ukraine, Kyiv in 1991, Senior researcher - in 1998, Doctor of Technical Sciences-in 2008, and Professor degree in 2019. The main areas of research - power supply systems, which are characterized by a wide range of changes in load parameters (primarily technological and transport purposes) in order to improve their energy, technical and economic efficiency and electromagnetic compatibility. He can be contacted at email: ol.nik.yurchenko@gmail.com. 